\title{
RELATIVE BRAUER GROUPS AND ÉTALE COHOMOLOGY
}

\author{
VIVEK SADHU
}

ABstRaCT. In this article, we construct a natural group homomorphism

$$
\theta: \operatorname{Br}(f) \rightarrow H_{e t}^{1}\left(S, f_{*} \mathcal{O}_{X}^{\times} / \mathcal{O}_{S}^{\times}\right)
$$

for a faithful affine map $f: X \rightarrow S$ of noetherian schemes. Here $\operatorname{Br}(f)$ denotes the relative Brauer group of $f$. We also prove $\operatorname{Br}(f)=0$ whenever $f: A \hookrightarrow B$ is a subintegral extension of noetherian $\mathbb{Q}$-algebras. Furthermore, we prove a relative version of Kummer's exact sequence.

\section{INTRODUCTION}

For a map $f: X \rightarrow S$ of schemes, we define the relative Brauer group $\operatorname{Br}(f)$ as the Grothendieck group of a certain relative category $A z\left(f^{*}\right)$, i.e., $\operatorname{Br}(f):=K_{0}\left(A z\left(f^{*}\right)\right.$ )(see section 2). This $\operatorname{Br}(f)$ fits into a natural exact sequence

$$
\operatorname{Pic}(S) \rightarrow \operatorname{Pic}(X) \rightarrow \operatorname{Br}(f) \rightarrow \operatorname{Br}(S) \rightarrow \operatorname{Br}(X) .
$$

Classically, the relative Brauer group is just the kernel of the natural map $\operatorname{Br}(S) \stackrel{f^{*}}{\rightarrow}$ $\operatorname{Br}(X)$ and is denoted by $\operatorname{Br}(X / S)$. Note that the relative Brauer group $\operatorname{Br}(f)$ is different from the classical one $\operatorname{Br}(X / S)$. But in the case of field extension, i.e., if $f: L \hookrightarrow K$ is a field extension then $\operatorname{Br}(f)$ is isomorphic to $\operatorname{Br}(K \mid L)$. The details related to $\operatorname{Br}(K \mid L)$ can be found in [7, 8], 9]. In general, we have a natural map $\operatorname{Br}(f) \rightarrow \operatorname{Br}(X / S)$ and, this map is an isomorphism if and only if $\operatorname{Pic}(S) \rightarrow \operatorname{Pic}(X)$ is surjective. .

Now assume that $f: X \rightarrow S$ is a faithful affine map of schemes, i.e., affine and the structure map $\mathcal{O}_{S} \rightarrow f_{*} \mathcal{O}_{X}$ is injective. We define

$$
\operatorname{Br}^{\prime}(f):=H_{e t}^{1}\left(S, f_{*} \mathcal{O}_{X}^{\times} / \mathcal{O}_{S}^{\times}\right) .
$$

If $f: L \hookrightarrow K$ is a finite field extension then $\operatorname{Br}(f) \cong \operatorname{Br}^{\prime}(f)$ (see Lemma 3.1). $\operatorname{But} \operatorname{Br}(f)$ and $\operatorname{Br}^{\prime}(f)$ are different in general (see Example 3.3). This is why we prefer to call the latter group as a relative cohomological Brauer group. One of the goals of this article is to relate $\operatorname{Br}(f)$ and $\operatorname{Br}^{\prime}(f)$. We prove the following (see Theorem 4.12):

Date: May 5, 2020.

Key words and phrases. Relative Brauer groups, Subintegral map, Kummer's sequence.

Author was supported by SERB-DST MATRICS grant MTR/2018/000283. 
Theorem 1.1. Let $f: X \rightarrow S$ be a faithful affine map of noetherian schemes. Then there is a natural group homomorphism $\theta: \operatorname{Br}(f) \rightarrow \operatorname{Br}^{\prime}(f):=H_{e t}^{1}\left(S, f_{*} \mathcal{O}_{X}^{\times} / \mathcal{O}_{S}^{\times}\right)$.

We give an outline of the proof of Theorem 1.1. It is known that there is a natural isomorphism $\check{H}_{e t}^{1}(S, \mathcal{F}) \stackrel{\cong}{\rightarrow} H_{e t}^{1}(S, \mathcal{F})$ for every abelian sheaf $\mathcal{F}$. Here $\check{H}_{e t}^{1}(S, \mathcal{F})$ denotes the first étale Čech cohomology group associated to $\mathcal{F}$. So we can restrict the target of the map $\theta$ to the first étale Cech cohomology group associated to $\left(f_{*} \mathcal{O}_{X}^{\times} / \mathcal{O}_{S}^{\times}\right)_{e t}$. Let $\mathcal{P} i c_{e t}^{f}$ be the étale sheaf associated to the presheaf $\operatorname{Pic}^{f}$ on $S_{e t}$, defined as $\operatorname{Pic}^{f}(U)=\operatorname{Pic}\left(f_{U}\right)$, where $f_{U}: X \times_{S} U \rightarrow U$ and the group Pic $\left(f_{U}\right)$ is generated by pairs $\left(L_{1}, L_{2}\right)$ of line bundles on $U$ together with an isomorphism $f_{U}^{*} L_{1} \cong f_{U}^{*} L_{2}$ of $\mathcal{O}_{X \times{ }_{S} U}$-modules with suitable relations (for details, see [19]). There is a natural isomorphism $\left(f_{*} \mathcal{O}_{X}^{\times} / \mathcal{O}_{S}^{\times}\right)_{e t} \cong$ $\mathcal{P} i c_{e t}^{f}$ (see Lemma 4.11). Some well-known facts pertaining to (pre)-sheaf torsors and the first étale Čech cohomology group allow us to restrict further the target of the map $\theta$ to the set of isomorphism classes of $\mathrm{Pic}^{f}$-torsors. Therefore, the problem boils down to defining a $\operatorname{Pic}^{f}$-torsor associated to each element of $\operatorname{Br}(f)$. Given an Azumaya algebra $A$ over a scheme $X$, one can associate a fibered category $F_{A}$ over $X_{\text {et }}$ which in fact defines an element in $H_{e t}^{2}\left(X, \mathcal{O}_{X}^{\times}\right)$. More explicitly, $A \mapsto F_{A}$ defines a natural map $\operatorname{Br}(X) \rightarrow H_{e t}^{2}\left(X, \mathcal{O}_{X}^{\times}\right)$(see [10], [12]). We follow a similar approach to prove our desired assertion. Note that the relative $\operatorname{Br}(f)$ is the abelian group generated by isomorphism classes of objects in $A z\left(f^{*}\right)$ modulo certain relations. An object of $A z\left(f^{*}\right)$ is a triple $\left(A_{1}, \alpha, A_{2}\right)$, where $A_{1}, A_{2}$ are Azumaya algebras over $S$ and $\alpha: f^{*} A_{1} \cong f^{*} A_{2}$ is an isomorphism in a suitable category. So we have the fibered categories $F_{A_{1}}$ and $F_{A_{2}}$ associated to $A_{1}$ and $A_{2}$. By using the categories $F_{A_{1}}$ and $F_{A_{2}}$, we define a relative category $G_{A}$ over $S_{e t}$ associated to $A:=\left(A_{1}, \alpha, A_{2}\right)$. Further, using the category $G_{A}$, we construct a $\mathrm{Pic}^{f}$-torsor associated to $A:=\left(A_{1}, \alpha, A_{2}\right)$ (see (4.8) and (4.9)). A significant portion of section 4 is dedicated to the construction of such a presheaf torsor and proving its required properties.

Next, we discuss the relative Brauer group of subintegral extensions. We say that an extension $A \hookrightarrow B$ is subintegral if $B$ is integral over $A$ and $\operatorname{Spec}(B) \rightarrow \operatorname{Spec}(A)$ is a bijection inducing isomorphisms on all residue fields. For example, $\mathbb{C}\left[t^{2}, t^{3}\right] \hookrightarrow \mathbb{C}[t]$ is a subintegral extension. We show that if $f: A \hookrightarrow B$ is a subintegral extension of noetherian $\mathbb{Q}$-algebras then the induced map $\operatorname{Br}(A) \stackrel{f^{*}}{\rightarrow} \operatorname{Br}(B)$ is an isomorphism and $\operatorname{Br}(f)=0$ (see Theorem 5.1).

We further study the Kummer exact sequence in the relative setting. Write $\mathcal{I}_{\text {et }}$ for the étale sheaf $f_{*} \mathcal{O}_{X}^{\times} / \mathcal{O}_{S}^{\times}$. Let $\mu_{n}^{f}$ denotes the kernel of $\mathcal{I}_{\text {et }} \stackrel{n}{\rightarrow} \mathcal{I}_{\text {et }}$. We prove that if $f: X \rightarrow S$ is a faithful finite map of schemes (i.e. finite and the structure map $\mathcal{O}_{S} \rightarrow f_{*} \mathcal{O}_{X}$ is 
injective) and characteristic of $k(s)$ does not divide $n$ for any $s \in S$ then the sequence

$$
0 \rightarrow \mu_{n}^{f} \rightarrow \mathcal{I}_{\text {et }} \stackrel{n}{\rightarrow} \mathcal{I}_{\text {et }} \rightarrow 0
$$

of étale sheaves is exact (see Proposition 6.2). As an application, we obtain $H_{e t}^{0}\left(S, \mu_{n}^{f}\right) \cong$ ${ }_{n} \operatorname{Pic}(f)$ and the following short exact sequence (see Theorem 6.3)

$$
0 \rightarrow \operatorname{Pic}(f) \otimes \mathbb{Z} / \mathrm{n} \mathbb{Z} \rightarrow H_{e t}^{1}\left(S, \mu_{n}^{f}\right) \rightarrow{ }_{n} H_{e t}^{1}\left(S, \mathcal{I}_{e t}\right) \rightarrow 0,
$$

where Pic $(f)$ is the relative Picard group of $f$ (see [19]). We also show that if $f: A \hookrightarrow B$ is a finite subintegral extension of noetherian $\mathbb{Q}$-algebras then $H_{e t}^{i}\left(\operatorname{Spec}(A), \mu_{n}^{f}\right)=0$ for $i \geq 0$ (see Theorem 6.4).

Acknowledgements: The author is grateful to Charles Weibel for his helpful comments during the preparation of this article. He would also like to thank the referee for valuable comments and suggestions.

\section{Relative Brauer groups}

In this section, we define the notion of relative Brauer groups. The most of the material in this section was developed in [1, 2], 3], [4, [6] by various authors.

Recall that two Azumaya algebras $A$ and $A^{\prime}$ over a scheme $X$ are said to be similar if there exist locally free $\mathcal{O}_{X}$-modules $E$ and $E^{\prime}$, of finite rank over $\mathcal{O}_{X}$, such that

$$
A \otimes_{\mathcal{O}_{X}} \operatorname{End}(E) \cong A^{\prime} \otimes_{\mathcal{O}_{X}} \operatorname{End}\left(E^{\prime}\right) .
$$

The set of similar classes of Azumaya algebras on $X$ forms an abelian group under $\otimes_{\mathcal{O}_{X}}$, which is known as the Brauer group $\operatorname{Br}(X)$ of $X$. If $[A] \in \operatorname{Br}(X)$ then $[A]^{-1}=\left[A^{o p}\right]$, where $A^{o p}$ denotes the opposite algebra of $A$. The cohomological Brauer group of $X$ is $H_{e t}^{2}\left(X, \mathcal{O}_{X}^{\times}\right)$and is denoted by $\operatorname{Br}^{\prime}(X)$. The Picard group of $X$ is denoted by $\operatorname{Pic}(X)$.

Given two Azumaya algebras $A$ and $B$ over $X$, let $\Delta(A, B)$ (resp. $\tilde{\Delta}(A, B))$ be the set consisting of all triples $(P, u, Q)$ with $P, Q$ are (resp. self dual) locally free $\mathcal{O}_{X}$-modules of finite rank and

$$
u: A \otimes \operatorname{End}(P) \rightarrow B \otimes \operatorname{End}(Q)
$$

is an isomorphism of algebras. We define an equivalence relation $\sim$ on $\Delta(A, B)$ by $(P, u, Q) \sim\left(P^{\prime}, u^{\prime}, Q^{\prime}\right)$ if and only if there exist locally free $\mathcal{O}_{X}$-modules $E$ and $E^{\prime}$ of finite rank over $X$ and $P \otimes E \cong P^{\prime} \otimes E^{\prime}, Q \otimes E \cong Q^{\prime} \otimes E^{\prime}$. By considering $E$ and $E^{\prime}$ are to be self dual locally free $\mathcal{O}_{X}$-modules, one can check that $\sim$ is an equivalence relation on $\tilde{\Delta}(A, B)$ as well.

Remark 2.1. Note that $u$ and $u^{\prime}$ do not play any role for the equivalence relation $\sim$ on $\Delta(A, B)$ (resp. $\tilde{\Delta}(A, B))$. In fact $(P, u, Q)$ and $(P, v, Q)$ both lie in a same class 
whenever $u, v$ are possibly distinct $\mathcal{O}_{X}$-algebra isomorphisms between $A \otimes \operatorname{End}(P)$ and $B \otimes \operatorname{End}(Q)$.

The category $A z(X) . A z(X)$ is the category whose objects are the Azumaya algebras over a scheme $X$ and the set of morphisms between two objects $A$ and $B$ is defined by

$$
\operatorname{Hom}_{A z(X)}(A, B):=\Delta(A, B) / \sim \text {. }
$$

Let $\phi_{1}=\left[\left(P_{1}, u_{1}, Q_{1}\right)\right] \in \operatorname{Hom}_{A z(X)}(A, B), \phi_{2}=\left[\left(P_{2}, u_{2}, Q_{2}\right)\right] \in \operatorname{Hom}_{A z(X)}(B, C)$. Then we define the composition $\phi_{2} \phi_{1}:=\left[\left(P_{1} \otimes P_{2}, u, Q_{1} \otimes Q_{2}\right)\right]$ with $u: A \otimes \operatorname{End}\left(P_{1} \otimes P_{2}\right) \cong$ $A \otimes \operatorname{End}\left(P_{1}\right) \otimes \operatorname{End}\left(P_{2}\right) \stackrel{u_{1}}{\cong} B \otimes \operatorname{End}\left(Q_{1}\right) \otimes \operatorname{End}\left(P_{2}\right) \stackrel{u_{2}}{\cong} C \otimes \operatorname{End}\left(Q_{2}\right) \otimes \operatorname{End}\left(Q_{1}\right) \cong$ $C \otimes \operatorname{End}\left(Q_{1} \otimes Q_{2}\right)$. One can easily check that this composition is independent of the chosen representatives of $\phi_{1}$ and $\phi_{2}$. If $\phi=[(P, u, Q)] \in \operatorname{Hom}_{A z(X)}(A, B)$ then $\phi$ is an isomorphism with inverse $\phi^{-1}=\left[\left(Q, u^{-1}, P\right)\right]$ (see Remark 5.7 of [] ). The tensor product $\otimes$ on $\mathcal{O}_{X}$-algebras defines a product on $A z(X)$.

The category $\Omega A z(X) . \Omega A z(X)$ is the category consisting of all couples $(A, \phi)$ with $A \in A z(X)$ and $\phi$ is an automorphism in $A z(X)$. A morphism $(A, \phi) \rightarrow(B, \varphi)$ is a morphism $h: A \rightarrow B$ in $A z(X)$ such that $\varphi h=h \phi$. Note that $\Omega A z(X)$ is a category with product and composition in the sense of [3]. We refer to 5.12 of [6] for product and composition rules in $\Omega A z(X)$.

We now state a result from [6].

Lemma 2.2. Let $X$ be a scheme. Then there are natural isomorphisms of abelian groups

(1) $K_{0}(A z(X)) \stackrel{\cong}{\rightarrow} \operatorname{Br}(X)$;

(2) $K_{0}(\Omega A z(X)) \stackrel{\cong}{\rightarrow} \operatorname{Pic}(X)$.

Proof. We get the assertions by applying Theorem 5.9 and Theorem 5.13 of [6] to the category of $\mathcal{O}_{X}$-modules.

For a map $f: X \rightarrow S$ of schemes, we have the base change functor $f^{*}: A z(S) \rightarrow$ $A z(X)$.

The category $A z\left(f^{*}\right) . A z\left(f^{*}\right)$ is the category consisting of all triples $(A, \alpha, B)$ where $A, B \in A z(S)$ and $\alpha: f^{*}(A) \rightarrow f^{*}(B)$ is an isomorphism in $A z(X)$. Here $\alpha=[(P, u, Q)]$ with $P, Q$ are locally free $\mathcal{O}_{X}$-modules of finite rank and $u: f^{*} A \otimes \operatorname{End}(P) \cong f^{*} B \otimes$ $\operatorname{End}(Q)$ (i.e. $f^{*} A$ is similar to $\left.f^{*} B\right)$. A morphism $(A, \alpha, B) \rightarrow\left(A^{\prime}, \alpha^{\prime}, B^{\prime}\right)$ is a pair $(u, v)$, where $u: A \rightarrow A^{\prime}$ and $v: B \rightarrow B^{\prime}$ are morphisms in $A z(S)$ such that $\alpha^{\prime} f^{*} u=f^{*} v \alpha$. We refer to 5.17 of [6] for product and composition rules in $A z\left(f^{*}\right)$.

Following Bass [3], we define the relative Brauer group $\operatorname{Br}(f)$ of $f$ to be the Grothendieck group $K_{0}\left(A z\left(f^{*}\right)\right)$, i.e., the abelian group generated by $\left[\left(A_{1}, \alpha, A_{2}\right)\right]$, where $\left(A_{1}, \alpha, A_{2}\right) \in A z\left(f^{*}\right)$, and with the following relations: 
(1) $\left[\left(A_{1}, \alpha, A_{2}\right)\right]+\left[\left(A_{1}^{\prime}, \alpha^{\prime}, A_{2}^{\prime}\right)\right]=\left[\left(A_{1} \otimes A_{1}^{\prime}, \alpha \otimes \alpha^{\prime}, A_{2} \otimes A_{2}^{\prime}\right)\right]$;

(2) $\left[\left(A_{1}, \alpha, A_{2}\right)\right]+\left[\left(A_{2}, \beta, A_{3}\right)\right]=\left[\left(A_{1}, \beta \alpha, A_{3}\right)\right]$.

Remark 2.3. By $(2),[(A, 1, A)]=0$ for any $A \in A z(S)$. Then by using (1), we get that every element of $\operatorname{Br}(f)$ has the form $\left[\left(A, \alpha, \operatorname{End}_{\mathcal{O}_{S}}(B)\right)\right]$, where $A$ and $B$ are in $A z(S)$. For general details, see Remark 1.3 in Chapter 1 of [3].

By Theorem 5.18 of [6] (more precisely, Example $5.19(2)$ of [6]), there is a natural exact sequence of abelian groups for each $f: X \rightarrow S$,

$$
\operatorname{Pic}(S) \stackrel{f^{*}}{\rightarrow} \operatorname{Pic}(X) \stackrel{\partial}{\rightarrow} \operatorname{Br}(f) \stackrel{\varrho}{\rightarrow} \operatorname{Br}(S) \stackrel{f^{*}}{\rightarrow} \operatorname{Br}(X) .
$$

Here $\varrho\left(\left[\left(A_{1}, \alpha, A_{2}\right)\right]\right)=A_{1} \otimes A_{2}^{o p}$ and the map $\partial$ is defined as follows. By Lemma $2.2(2)$, we can identify Pic $(X)$ with $K_{0}(\Omega A z(X))$. Let $A z^{\prime}(X)$ denote the full subcategory of $A z(X)$ whose objects are all $f^{*} A, A \in A z(S)$. The functor $f^{*}: A z(S) \rightarrow A z(X)$ is cofinal (see Proposition 5.15 of [6]). So, $A z^{\prime}(X)$ is a cofinal subcategory of $A z(X)$. We refer to p. 19 of [3] for general details related to cofinal functors and cofinal subcategories. Now the Theorem 3.1(b) of [3] says that there is an isomorphism $\kappa: K_{0}\left(\Omega A z^{\prime}(X)\right) \rightarrow$ $K_{0}(\Omega A z(X))$. We also have a natural homomorphism $\partial_{1}: K_{0}\left(\Omega A z^{\prime}(X)\right) \rightarrow K_{0}\left(A z\left(f^{*}\right)\right)$, sending $\left[\left(f^{*} A, \alpha\right)\right]$ to $[(A, \alpha, A)]$. Hence we define $\partial: K_{0}(\Omega A z(X)) \rightarrow K_{0}\left(A z\left(f^{*}\right)\right)$ as $\partial_{1} \kappa^{-1}$.

Remark 2.4. Suppose that $S=\operatorname{Spec}(\mathbb{Z})$, i.e., $f: X \rightarrow \operatorname{Spec}(\mathbb{Z})$. By Proposition 4.2 of $[20, \operatorname{Br}(\mathbb{Z})=0$. Then the sequence (2.1) implies that $\operatorname{Br}(f) \cong \operatorname{Pic}(X)$.

Next, we define a slight variant of the group $\operatorname{Br}(f)$ which will be used in the section 4.

The categories $\tilde{A} z(X)$ and $\tilde{A} z\left(f^{*}\right) . \tilde{A} z(X)$ is the category whose objects are the Azumaya algebras over a scheme $X$ and the set of morphisms between two objects $A$ and $B$ is defined by

$$
\operatorname{Hom}_{A z(X)}(A, B):=\tilde{\Delta}(A, B) / \sim .
$$

$\tilde{A} z\left(f^{*}\right)$ is the category consisting of all triples $(A, \alpha, B)$ where $A, B \in \tilde{A} z(S)$ and $\alpha$ : $f^{*}(A) \rightarrow f^{*}(B)$ is an isomorphism in $\tilde{A} z(X)$. A morphism $(A, \alpha, B) \rightarrow\left(A^{\prime}, \alpha^{\prime}, B^{\prime}\right)$ is a pair $(u, v)$, where $u: A \rightarrow A^{\prime}$ and $v: B \rightarrow B^{\prime}$ are morphisms in $\tilde{A} z(S)$ such that $\alpha^{\prime} f^{*} u=f^{*} v \alpha$. One can define a product and composition on $\tilde{A} z(X)\left(\operatorname{resp} . \tilde{A} z\left(f^{*}\right)\right)$ in a similar way as it is defined on $A z(X)$ (resp. $\left.A z\left(f^{*}\right)\right)$.

We define $\tilde{\operatorname{Br}}(f):=K_{0}\left(\tilde{A} z\left(f^{*}\right)\right)$. Given $A:=\left(A_{1}, \alpha, A_{2}\right) \in A z\left(f^{*}\right)$ with $\alpha=[(P, u, Q)]$, let $\mathbb{E n d}(A):=\left(\operatorname{End}_{\mathcal{O}_{S}}\left(A_{1}\right), \operatorname{End}(\alpha), \operatorname{End}_{\mathcal{O}_{S}}\left(A_{2}\right)\right)$, where

$$
\operatorname{End}(\alpha):=\left[\left(\operatorname{End}_{\mathcal{O}_{X}}(P), \operatorname{End}(u), \operatorname{End}_{\mathcal{O}_{X}}(Q)\right)\right]
$$


with

$$
\begin{aligned}
\operatorname{End}(u): f^{*} \operatorname{End}_{\mathcal{O}_{S}}\left(A_{1}\right) \otimes \operatorname{End}(\operatorname{End}(P)) & \cong \operatorname{End}_{\mathcal{O}_{X}}\left(f^{*} A_{1} \otimes \operatorname{End}(P)\right) \\
& \cong \operatorname{End}_{\mathcal{O}_{X}}\left(f^{*} A_{2} \otimes \operatorname{End}(Q)\right)(\text { using } u) \\
& \cong f^{*} \operatorname{End}_{\mathcal{O}_{S}}\left(A_{2}\right) \otimes \operatorname{End}(\operatorname{End}(Q)) .
\end{aligned}
$$

Observe that $\left(\operatorname{End}_{\mathcal{O}_{X}}(P), \operatorname{End}(u), \operatorname{End}_{\mathcal{O}_{X}}(Q)\right) \in \tilde{\Delta}\left(f^{*} \operatorname{End}_{\mathcal{O}_{S}}\left(A_{1}\right), f^{*} \operatorname{End}_{\mathcal{O}_{S}}\left(A_{2}\right)\right)$. So $\mathbb{E} n d(A) \in \tilde{A} z\left(f^{*}\right)$. We define a map $\mathbb{E} n d: o b\left(A z\left(f^{*}\right)\right) \rightarrow \tilde{\operatorname{Br}}(f)$ by $A \mapsto[\mathbb{E} n d(A)]$. The following facts are easy to verify:

(1) If $A:=\left(A_{1}, \alpha, A_{2}\right) \cong B:=\left(B_{1}, \beta, B_{2}\right)$ in $A z\left(f^{*}\right)$ then $[\mathbb{E} n d(A)] \cong[\mathbb{E} n d(B)]$ in $\tilde{\operatorname{Br}}(f)$.

(2) $\left[\mathbb{E} n d\left(A_{1} \otimes A_{1}^{\prime}, \alpha \otimes \alpha^{\prime}, A_{2} \otimes A_{2}^{\prime}\right)\right]=\left[\mathbb{E} n d\left(A_{1}, \alpha, A_{2}\right)\right]+\left[\mathbb{E} n d\left(A_{1}^{\prime}, \alpha^{\prime}, A_{2}^{\prime}\right)\right]$, for every pair of objects $\left(A_{1}, \alpha, A_{2}\right),\left(A_{1}^{\prime}, \alpha^{\prime}, A_{2}^{\prime}\right)$ in $A z\left(f^{*}\right)$.

(3) $\left[\mathbb{E} n d\left(A_{1}, \beta \alpha, A_{3}\right)\right]=\left[\mathbb{E} n d\left(A_{1}, \alpha, A_{2}\right)\right]+\left[\mathbb{E} n d\left(A_{2}, \beta, A_{3}\right)\right]$, for every pair of objects $\left(A_{1}, \alpha, A_{2}\right),\left(A_{2}, \beta, A_{3}\right)$ in $A z\left(f^{*}\right)$.

Therefore, the map $\mathbb{E} n d$ induces a natural group homomorphism

$$
\Upsilon: \operatorname{Br}(f) \rightarrow \tilde{\operatorname{Br}}(f)
$$

sending $\left[\left(A_{1}, \alpha, A_{2}\right)\right]$ to $\left[\mathbb{E} n d\left(\left(A_{1}, \alpha, A_{2}\right)\right)\right]$.

\section{Relative Cohomological Brauer Groups}

Let $f: L \hookrightarrow K$ be a field extension. Then the relative Brauer group in the classical sense is just the kernel of the natural map $\operatorname{Br}(L) \rightarrow \operatorname{Br}(K)$ and it was denoted by $\operatorname{Br}(K \mid L)$ (for details, see [7], 8], [9]). We begin with the following observation.

Lemma 3.1. If $f: L \hookrightarrow K$ is a finite field extension then $H_{\text {et }}^{1}\left(\operatorname{Spec}(L), f_{*} \mathcal{O}_{K}^{\times} / \mathcal{O}_{L}^{\times}\right) \cong$ $\operatorname{Br}(K \mid L)$.

Proof. For a field $F, H_{e t}^{2}\left(F, \mathcal{O}_{F}^{\times}\right) \cong B r(F)$ and $H_{e t}^{1}\left(F, \mathcal{O}_{F}^{\times}\right) \cong \operatorname{Pic}(F)=0$. Then (by using the exact sequence (2.1))

$$
\begin{aligned}
H_{e t}^{1}\left(\operatorname{Spec}(L), f_{*} \mathcal{O}_{K}^{\times} / \mathcal{O}_{L}^{\times}\right) & \cong \operatorname{ker}\left[H_{e t}^{2}\left(L, \mathcal{O}_{L}^{\times}\right) \rightarrow H_{e t}^{2}\left(K, \mathcal{O}_{K}^{\times}\right)\right] \\
& \cong \operatorname{ker}[\operatorname{Br}(L) \rightarrow \operatorname{Br}(K)] \\
& \cong \operatorname{Br}(K \mid L)
\end{aligned}
$$

Motivated by the above lemma, we define

$$
\operatorname{Br}^{\prime}(f):=H_{e t}^{1}\left(S, f_{*} \mathcal{O}_{X}^{\times} / \mathcal{O}_{S}^{\times}\right)
$$


for a faithful affine map $f: X \rightarrow S$ of schemes. We call it relative cohomological Brauer group of $f$.

Example 3.2. Suppose that $f: X \rightarrow S$ is faithful finite, i.e., finite and the structure map $\mathcal{O}_{S} \rightarrow f_{*} \mathcal{O}_{X}$ is injective and $S=\operatorname{Spec}(A)$, where $A$ is a hensel local ring. Then $X=\operatorname{Spec}(B)$ and $B$ is a finite product of hensel local rings. In this situation, $\operatorname{Br}(S) \cong$ $H_{e t}^{2}\left(S, \mathcal{O}_{S}^{\times}\right)$and $\operatorname{Br}(X) \hookrightarrow H_{e t}^{2}\left(X, \mathcal{O}_{X}^{\times}\right)$by Corollary 2.12 of [12]. Therefore, by a similar argument as Lemma $3.1, \operatorname{Br}(f) \cong \operatorname{Br}^{\prime}(f)$.

Example 3.3. In general, $\operatorname{Br}(f)$ and $\operatorname{Br}^{\prime}(f)$ are not isomorphic for any faithful affine $f: X \rightarrow S$. For example, let $S=\operatorname{Spec}(A)$, where $A$ is a strictly hensel local ring. Then $X=\operatorname{Spec}(B)$, and $\operatorname{Br}^{\prime}(f)=0$ because the higher cohomology vanishes for a strictly henselian ring. We also have $\operatorname{Br}(S)=0$. Therefore $\operatorname{Br}(f) \cong \operatorname{Pic}(B)$ by (2.1). In particular, one can consider $\mathbb{C} \subset \mathbb{C}\left[t^{2}, t^{3}\right]$.

Example 3.4. Suppose that $f: X \rightarrow S$ is faithful finite and $S=\operatorname{Spec}(A)$, where $A$ is a local ring. Then $X=\operatorname{Spec}(B)$ and $B$ is a semilocal ring because $B$ is finite over a local ring. By (2.1),

$$
0 \rightarrow \operatorname{Br}(f) \rightarrow \operatorname{Br}(A) \rightarrow \operatorname{Br}(B)
$$

is exact and $\operatorname{Br}(f) \hookrightarrow \operatorname{Br}^{\prime}(f)$. We know that if a scheme $X$ has only finitely many connected components then $\operatorname{Br}(X)$ is torsion (see Proposition 2.7 of [12]). Therefore $\operatorname{Br}(f)$ is torsion. By Theorem 1.1 of [5], $\operatorname{Br}(A) \cong H_{e t}^{2}\left(A, \mathcal{O}_{A}^{\times}\right)_{\text {tor }}$ and same holds for $B$. In fact, we get $\operatorname{Br}(f) \cong H_{\text {et }}^{1}\left(S, f_{*} \mathcal{O}_{X}^{\times} / \mathcal{O}_{S}^{\times}\right)_{\text {tor }}$.

Example 3.5. The group $\operatorname{Br}(f)$ is not always torsion. For example, consider $f: X=$ $\operatorname{Spec}\left(\mathbb{Q}\left[t^{2}-t, t^{3}-t^{2}\right]\right) \rightarrow S=\operatorname{Spec}(\mathbb{Q})$. Then $\operatorname{Pic}(X) \cong \mathbb{Q}^{\times}$. By (2.1), we get that $\operatorname{Br}(f)$ contains the torsion free subgroup $\mathbb{Q}^{\times}$.

\section{MAIN ThEOREM}

The main goal of this section is to construct a natural group homomorphism $\operatorname{Br}(f) \rightarrow$ $H_{e t}^{1}\left(S, f_{*} \mathcal{O}_{X}^{\times} / \mathcal{O}_{S}^{\times}\right)$. Throughout this section, $f: X \rightarrow S$ assumed to be a faithful affine map of schemes and $f_{U}$ always denotes the map $X \times{ }_{S} U \rightarrow U$.

The category $F_{A}$. Given an Azumaya algebra $A$ on $X$ one can associate a category $F_{A}$ over $X_{e t}$ as follows (see p. 145 of [12]). For an étale map $j: U \rightarrow X$, an object of $F_{A}(U)$ is a pair $(E, \tau)$, where $E$ is a locally free $\mathcal{O}_{U}$-module of finite rank and $\tau$ is an isomorphism $\operatorname{End}(E) \cong j^{*} A$; a morphism $(E, \tau) \rightarrow\left(E^{\prime}, \tau^{\prime}\right)$ is an isomorphism $E \rightarrow E^{\prime}$ such that the obvious diagram 


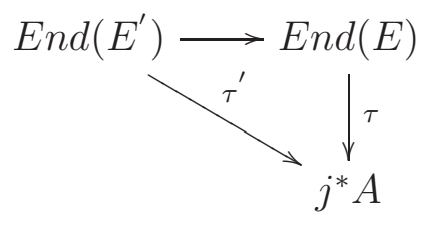

commutes.

Notation: Given a sheaf $\mathcal{F}$ of $\mathcal{O}_{X}$-modules, we write $\mathcal{F}^{\vee}$ for the dual of $\mathcal{F}$.

The category $G_{A}$. Given an object $A:=\left(A_{1}, \alpha, A_{2}\right)$ in $\tilde{A} z\left(f^{*}\right)$ (or in $A z\left(f^{*}\right)$ ), we can associate a category $G_{A}$ over $S_{e t}$ as follows. For an étale map $j: U \rightarrow S$, an object of $G_{A}(U)$ is a triple $\left(\left(E_{1}, \tau_{1}\right), \nu,\left(E_{2}, \tau_{2}\right)\right)$, where $\left(E_{i}, \tau_{i}\right) \in F_{A_{i}}(U)$ for $i=1,2$ and $\nu$ is an isomorphism $f_{U}^{*} E_{1} \otimes\left(f_{U}^{*} E_{2}\right)^{\vee} \cong\left(f_{U}^{*} E_{1}\right)^{\vee} \otimes f_{U}^{*} E_{2}$ of $\mathcal{O}_{X \times_{S} U}$-modules. Here $F_{A_{i}}$ are the categories associated to $A_{i}$. Similarly, a morphism $\left(\left(E_{1}, \tau_{1}\right), \nu,\left(E_{2}, \tau_{2}\right)\right) \rightarrow$ $\left(\left(E_{1}^{\prime}, \tau_{1}^{\prime}\right), \nu^{\prime},\left(E_{2}^{\prime}, \tau_{2}^{\prime}\right)\right)$ is a pair of isomorphisms $E_{1} \rightarrow E_{1}^{\prime}$ and $E_{2} \rightarrow E_{2}^{\prime}$ such that the diagram like (4.1) and

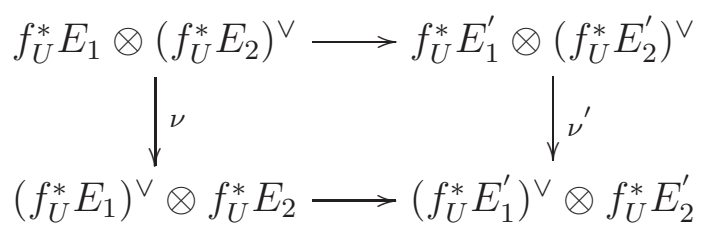

commute.

Let $\left[G_{A}(U)\right]$ be the set of all isomorphism classes of objects of $G_{A}(U)$. We write $\left[\left(\left(E_{1}, \tau_{1}\right), \nu,\left(E_{2}, \tau_{2}\right)\right)\right]$ for the isomorphism class of $\left(\left(E_{1}, \tau_{1}\right), \nu,\left(E_{2}, \tau_{2}\right)\right)$. Then the assignment

$$
U \mapsto\left[G_{A}(U)\right]
$$

is a presheaf of sets on $S_{e t}$, where the restriction maps are given by pullbacks. It is denoted by $\left[G_{A}\right]$.

Presheaf torsors. Let $\mathcal{C}$ be a site. Let $\mathcal{G}$ be a presheaf of groups on $\mathcal{C}$. A $\mathcal{G}$-torsor is a presheaf of sets $\mathcal{F}$ on $\mathcal{C}$ equipped with an action $\rho: \mathcal{G} \times \mathcal{F} \rightarrow \mathcal{F}$ such that

(1) the action $\rho(U): \mathcal{G}(U) \times \mathcal{F}(U) \rightarrow \mathcal{F}(U)$ is simply transitive provided $\mathcal{F}(U)$ is nonempty.

(2) for every $U \in o b(\mathcal{C})$ there exists a covering $\left\{U_{i} \rightarrow U\right\}_{i \in I}$ of $U$ such that $\mathcal{F}\left(U_{i}\right)$ is nonempty for all $i$.

A morphism of $\mathcal{G}$-torsors $\mathcal{F} \rightarrow \mathcal{F}^{\prime}$ is just a morphism of presheaves of sets compatible with the $\mathcal{G}$-action. A trivial $\mathcal{G}$-torsor is the presheaf $\mathcal{G}$ with obvious left $\mathcal{G}$-action. Note that a morphism between $\mathcal{G}$-torsors is always an isomorphism. Moreover, a $\mathcal{G}$-torsor $\mathcal{F}$ is trivial if and only if $\Gamma(\mathcal{C}, \mathcal{F}) \neq \emptyset$ (see chapter 21 of [16]). 
Relative Picard groups. The relative Pic $(f)$ is the abelian group generated by $\left[L_{1}, \alpha, L_{2}\right]$, where the $L_{i}$ are line bundles on $S$ and $\alpha: f^{*} L_{1} \rightarrow f^{*} L_{2}$ is an isomorphism. The relations are:

(1) $\left[L_{1}, \alpha, L_{2}\right]+\left[L_{1}^{\prime}, \alpha^{\prime}, L_{2}^{\prime}\right]=\left[L_{1} \otimes L_{1}^{\prime}, \alpha \otimes \alpha^{\prime}, L_{2} \otimes L_{2}^{\prime}\right]$;

(2) $\left[L_{1}, \alpha, L_{2}\right]+\left[L_{2}, \beta, L_{3}\right]=\left[L_{1}, \beta \alpha, L_{3}\right]$;

(3) $\left[L_{1}, \alpha, L_{2}\right]=0$ if $\alpha=f^{*}\left(\alpha_{0}\right)$ for some $\alpha_{0}: L_{1} \cong L_{2}$.

This relative Picard group Pic $(f)$ fits into the following exact sequence

$$
1 \rightarrow \mathcal{O}^{\times}(S) \rightarrow \mathcal{O}^{\times}(X) \rightarrow \operatorname{Pic}(f) \rightarrow \operatorname{Pic}(S) \rightarrow \operatorname{Pic}(X) .
$$

Some relevant details and basic properties can be found in [18, [19].

Next, we define a group which is a slight variant of the group Pic $(f)$.

The group Pic $(f)$. The relative $\tilde{\mathrm{Pic}}(f)$ is the abelian group generated by $\left[L_{1}, \alpha, L_{2}\right]$, where the $L_{i}$ are line bundles on $S$ and $\alpha: f^{*} L_{1} \otimes f^{*} L_{2}^{-1} \rightarrow f^{*} L_{1}^{-1} \otimes f^{*} L_{2}$ is an isomorphism. The relations are similar to Pic $(f)$.

There is a natural group homomorphism

$$
\Psi: \operatorname{Pic}(f) \rightarrow \operatorname{Pic}(f)
$$

sending $\left[L_{1}, \alpha, L_{2}\right]$ to $\left[L_{1} \otimes L_{2}^{-1}, \alpha, L_{1}^{-1} \otimes L_{2}\right]$.

Let $\operatorname{Pic}^{f}$ be the étale presheaf on $S_{e t}$, defined as $\operatorname{Pic}^{f}(U)=\operatorname{Pic}\left(f_{U}\right)$. Similarly, we can define the étale presheaf $\tilde{\mathrm{Pic}}^{f}$ on $S_{e t}$.

Lemma 4.1. Let $V_{1}, V_{2}$ and $V_{3}$ be three locally free $\mathcal{O}_{X}$-modules of finite rank. Suppose that $\iota: V_{1} \otimes V_{3} \rightarrow V_{2} \otimes V_{3}$ is an isomorphism as an $\mathcal{O}_{X}$-module. Then there is an $\mathcal{O}_{X}$-module isomorphism $V_{1} \rightarrow V_{2}$.

Proof. We know that $\mathcal{F} \otimes_{E n d(\mathcal{F})} \mathcal{F}^{\vee} \cong \mathcal{O}_{X}$ for any locally free $\mathcal{O}_{X}$-module $\mathcal{F}$ of finite rank. Then we get $V_{1} \cong V_{1} \otimes V_{3} \otimes_{\operatorname{End}\left(V_{3}\right)} V_{3}^{\vee} \stackrel{\iota \otimes i d}{\rightarrow} V_{2} \otimes V_{3} \otimes_{\operatorname{End}\left(V_{3}\right)} V_{3}^{\vee} \stackrel{\cong}{\rightarrow} V_{2}$.

Lemma 4.2. Let $A:=\left(A_{1}, \alpha, A_{2}\right)$ be in $\tilde{A} z\left(f^{*}\right)\left(\right.$ or in $\left.A z\left(f^{*}\right)\right)$. Let $\left(\left(E_{1}, \tau_{1}\right), \nu,\left(E_{2}, \tau_{2}\right)\right)$, $\left(\left(E_{1}^{\prime}, \tau_{1}^{\prime}\right), \nu^{\prime},\left(E_{2}^{\prime}, \tau_{2}^{\prime}\right)\right)$ be any two objects in $G_{A}(U)$, where $U \in$ ob $\left(S_{e t}\right)$. Then

(1) we can construct a unique element of $\tilde{\mathrm{Pic}}^{f}(U)$. Call it $\left[L_{1}, \beta, L_{2}\right]$.

(2) $\left(\left(L_{1} \otimes E_{1}, \tau_{1} a_{1}\right), \beta \otimes \nu,\left(L_{2} \otimes E_{2}, \tau_{2} a_{2}\right)\right)$ defines an object in $G_{A}(U)$, where $a_{k}$ : $\operatorname{End}\left(E_{k} \otimes L_{k}\right) \cong \operatorname{End}\left(E_{k}\right)$ for $k=1,2$. Moreover, $\left(\left(L_{1} \otimes E_{1}, \tau_{1} a_{1}\right), \beta \otimes \nu,\left(L_{2} \otimes\right.\right.$ $\left.\left.E_{2}, \tau_{2} a_{2}\right)\right)$ is isomorphic to $\left(\left(E_{1}^{\prime}, \tau_{1}^{\prime}\right), \nu^{\prime},\left(E_{2}^{\prime}, \tau_{2}^{\prime}\right)\right)$ in $G_{A}(U)$.

Proof. For simplicity, we write $E\left(E_{k}\right)$ instead of $E n d\left(E_{k}\right)$ throughout the proof.

(1) By definition, for $k=1,2$, we have

$$
\tau_{k}: E\left(E_{k}\right) \cong j^{*} A_{k}, \tau_{k}^{\prime}: E\left(E_{k}^{\prime}\right) \cong j^{*} A_{k}
$$


where $j$ denotes the étale map $U \rightarrow S$. Then $\tau_{k}^{\prime}-1 \tau_{k}: E\left(E_{k}\right) \cong E\left(E_{k}^{\prime}\right)$. So, we can find unique invertible sheaves $L_{k}=E_{k}^{\prime} \otimes_{E\left(E_{k}\right)} E_{k}^{\vee}$ such that (see Lemma 4.3 of [6])

$$
v_{k}: L_{k} \otimes_{\mathcal{O}_{U}} E_{k}=\left(E_{k}^{\prime} \otimes_{E\left(E_{k}\right)} E_{k}^{\vee}\right) \otimes_{\mathcal{O}_{U}} E_{k} \cong E_{k}^{\prime} \otimes_{E\left(E_{k}\right)}\left(E_{k}^{\vee} \otimes_{\mathcal{O}_{U}} E_{k}\right) \cong E_{k}^{\prime} .
$$

Note that $v_{k}$ 's are $\left(E\left(E_{k}\right), \mathcal{O}_{U}\right)$-linear isomorphisms. By using $\nu, \nu^{\prime}$ and $v_{k}$, we obtain an $\mathcal{O}_{X \times{ }_{S} U}$-module isomorphism

$$
\begin{aligned}
& f_{U}^{*} L_{1} \otimes f_{U}^{*} L_{2}^{-1} \otimes f_{U}^{*} E_{1} \otimes\left(f_{U}^{*} E_{2}\right)^{\vee} \cong f_{U}^{*} E_{1}^{\prime} \otimes\left(f_{U}^{*} E_{2}^{\prime}\right)^{\vee} \cong\left(f_{U}^{*} E_{1}\right)^{\prime \vee} \otimes f_{U}^{*} E_{2}^{\prime} \\
& \cong f_{U}^{*} L_{1}^{-1} \otimes f_{U}^{*} L_{2} \otimes\left(f_{U}^{*} E_{1}\right)^{\vee} \otimes f_{U}^{*} E_{2} \cong f_{U}^{*} L_{1}^{-1} \otimes f_{U}^{*} L_{2} \otimes f_{U}^{*} E_{1} \otimes\left(f_{U}^{*} E_{2}\right)^{\vee} .
\end{aligned}
$$

Set

$$
\begin{gathered}
L=f_{U}^{*} L_{1} \otimes f_{U}^{*} L_{2}^{-1}, L^{\vee}=f_{U}^{*} L_{1}^{-1} \otimes f_{U}^{*} L_{2}, \\
E=f_{U}^{*} E_{1} \otimes\left(f_{U}^{*} E_{2}\right)^{\vee}, E^{\vee}=\left(f_{U}^{*} E_{1}\right)^{\vee} \otimes f_{U}^{*} E_{2}, \\
E^{\prime}=f_{U}^{*} E_{1}^{\prime} \otimes\left(f_{U}^{*} E_{2}^{\prime}\right)^{\vee},\left(E^{\prime}\right)^{\vee}=\left(f_{U}^{*} E_{1}^{\prime}\right)^{\vee} \otimes f_{U}^{*} E_{2}^{\prime} .
\end{gathered}
$$

Thus, we have

$$
L \otimes E \stackrel{\Gamma}{\rightarrow} E^{\prime} \stackrel{\nu^{\prime}}{\rightarrow}\left(E^{\prime}\right)^{\vee} \stackrel{\Gamma^{\vee}}{\rightarrow} L^{\vee} \otimes E^{\vee} \stackrel{1_{L} \vee \otimes \nu^{-1}}{\rightarrow} L^{\vee} \otimes E
$$

where $1_{L^{\vee}}$ denotes the identity map on $L^{\vee}$.

Now the Lemma 4.1 implies that there is an $\mathcal{O}_{X \times{ }_{S} U}$-module isomorphism $\beta: L \cong L^{\vee}$. More precisely, we get $\beta$ as follows:

$$
L \stackrel{a}{\rightarrow} L \otimes E \otimes_{E(E)} E^{\vee} \stackrel{\delta \otimes 1_{E^{\vee}}}{\longrightarrow} L^{\vee} \otimes E^{\vee} \otimes_{E(E)} E^{\vee} \stackrel{1_{L^{\vee}} \otimes \nu^{-1} \otimes 1_{E} \vee}{\longrightarrow} L^{\vee} \otimes E \otimes_{E(E)} E^{\vee} \stackrel{b}{\rightarrow} L^{\vee}
$$

Here $\delta=\Gamma^{\vee} \nu^{\prime} \Gamma$. Therefore, $\left[L_{1}, \beta, L_{2}\right] \in \operatorname{Pic}^{f}(U)$. This proves (1).

(2) Note that $\beta \otimes \nu$ gives an isomorphism between $L \otimes E$ and $L^{\vee} \otimes E^{\vee}$. Hence, clearly $\left(\left(L_{1} \otimes E_{1}, \tau_{1} a_{1}\right), \beta \otimes \nu,\left(L_{2} \otimes E_{2}, \tau_{2} a_{2}\right)\right)$ defines an object in $G_{A}(U)$, where $a_{k}$ : $E\left(E_{k} \otimes L_{k}\right) \cong E\left(E_{k}\right)$ for $k=1,2$.

Next, we shall show that $\left(v_{1}, v_{2}\right)$ defines an isomorphism between $\left(\left(L_{1} \otimes E_{1}, \tau_{1} a_{1}\right), \beta \otimes\right.$ $\left.\nu,\left(L_{2} \otimes E_{2}, \tau_{2} a_{2}\right)\right)$ and $\left(\left(E_{1}^{\prime}, \tau_{1}^{\prime}\right), \nu^{\prime},\left(E_{2}^{\prime}, \tau_{2}^{\prime}\right)\right)$ in $G_{A}(U)$. To prove $\left(v_{1}, v_{2}\right)$ is an isomorphism in $G_{A}(U)$, we have to check that the diagrams like (4.1) and (4.2) commute. To check these commutativity, we may assume that $U$ is affine.

We first check that the following diagram

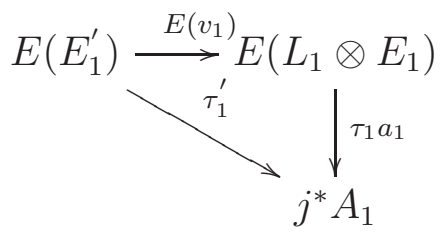

commutes. 
For $s \in E\left(E_{1}^{\prime}\right)$, we have $E\left(v_{1}\right)(s)=v_{1}^{-1} s v_{1}$. Note that $E\left(v_{1}\right)^{-1}=E\left(v_{1}^{-1}\right)$. Let $\tau=$ $\tau_{1}^{\prime-1} \tau_{1}$. So, it suffices to check that $E\left(v_{1}^{-1}\right) a_{1}^{-1}=\tau$, i.e., $\left(E\left(v_{1}^{-1}\right) a_{1}^{-1}\right)(t)=\tau(t)$ for all $t \in E\left(E_{1}\right)$. We can write $a_{1}^{-1}(t)=1_{L_{1}} \otimes t$. Moreover, $v_{1}$ is $E\left(E_{1}\right)$-linear map and $E_{1}^{\prime}$ is $E\left(E_{1}\right)$-module via $\tau$. Using these facts, we get $\left(\left(E\left(v_{1}^{-1}\right) a_{1}^{-1}\right)(t)\right)\left(e^{\prime}\right)=\tau(t)\left(e^{\prime}\right)$ for all $e^{\prime} \in E_{1}^{\prime}$. Hence the assertion.

Further, we show that the diagram like (4.2) commutes. By (4.5), we have the following commutative diagram

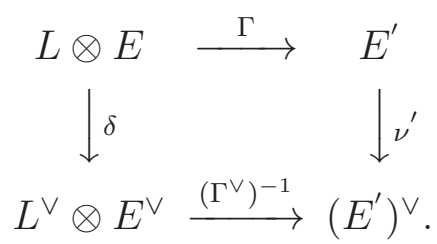

We claim that $\beta \otimes \nu=\delta$. Recall that $\beta=b\left(1_{L^{\vee}} \otimes \nu^{-1} \otimes 1_{E^{\vee}}\right)\left(\delta \otimes 1_{E^{\vee}}\right) a$. Then $\beta \otimes \nu=$ $(b \otimes \nu)\left(1_{L^{\vee}} \otimes \nu^{-1} \otimes 1_{E^{\vee}} \otimes 1_{E}\right)\left(\delta \otimes 1_{E^{\vee}} \otimes 1_{E}\right)\left(a \otimes 1_{E}\right)$. We get the following commutative diagram

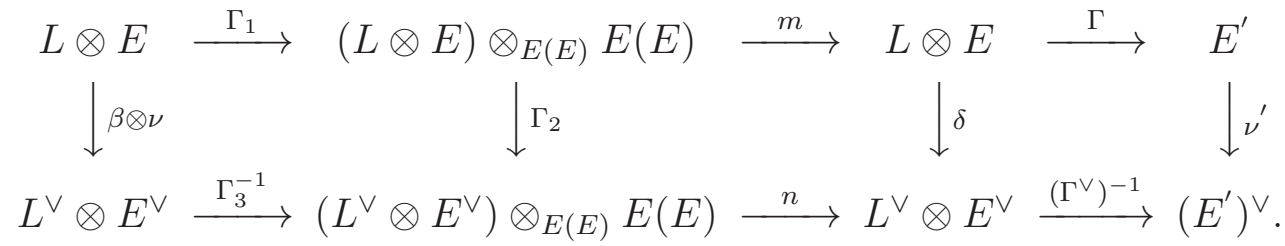

Here $\Gamma_{1}=a \otimes 1_{E}, \Gamma_{2}=\delta \otimes 1_{E^{\vee}} \otimes 1_{E}, \Gamma_{3}=(b \otimes \nu) \cdot\left(1_{L^{\vee}} \otimes \nu^{-1} \otimes 1_{E^{\vee}} \otimes 1_{E}\right)$ and $m, n$ are natural isomorphisms sending $x \otimes \tilde{e}$ to $x \tilde{e}$ for all $\tilde{e} \in E(E)$. One can easily check that $m \Gamma_{1}$ and $n \Gamma_{3}^{-1}$ both are identity isomorphisms. Hence $\beta \otimes \nu=\delta$. This completes the proof.

Given an étale map $U \rightarrow S$, we define a group action $\rho(U): \tilde{\operatorname{Pic}}^{f}(U) \times\left[G_{A}\right](U) \rightarrow$ $\left[G_{A}\right](U)$ by

$$
\left(\left[L_{1}, \beta, L_{2}\right],\left[\left(\left(E_{1}, \tau_{1}\right), \nu,\left(E_{2}, \tau_{2}\right)\right)\right]\right) \mapsto\left[\left(\left(L_{1} \otimes E_{1}, a_{1} \tau_{1}\right), \beta \otimes \nu,\left(L_{2} \otimes E_{2}, a_{2} \tau_{2}\right)\right] .\right.
$$

Here $L_{1}, L_{2}$ are line bundles on $U$ with an isomorphism $\beta: f_{U}^{*} L_{1} \otimes f_{U}^{*} L_{2}^{-1} \cong f_{U}^{*} L_{1}^{-1} \otimes f_{U}^{*} L_{2}$ and $a_{k}: \operatorname{End}\left(E_{k} \otimes L_{k}\right) \cong \operatorname{End}\left(E_{k}\right)$ for $k=1,2$.

Proposition 4.3. Let $A:=\left(A_{1}, \alpha, A_{2}\right)$ be in $\tilde{A} z\left(f^{*}\right)$ (or in $\left.A z\left(f^{*}\right)\right)$. Assume that $S$ is connected. Then $\left[G_{A}\right]$ is a $\tilde{\mathrm{Pic}}^{f}$-torsor.

Proof. We need to show that the following are true:

(1) the action $\rho(U)$ is simply transitive,

(2) for every $U \in o b\left(S_{e t}\right)$ there exist a étale covering $\left\{U_{i} \rightarrow U\right\}$ such that $\left[G_{A}\right]\left(U_{i}\right) \neq$ $\emptyset$ for all $i$. 
Let $\left[\left(E_{1}, \tau_{1}\right), \nu,\left(E_{2}, \tau_{2}\right)\right],\left[\left(E_{1}^{\prime}, \tau_{1}^{\prime}\right), \nu^{\prime},\left(E_{2}^{\prime}, \tau_{2}^{\prime}\right)\right] \in\left[G_{A}\right](U)$. Then by Lemma 4.2, we can construct a unique $\left[L_{1}, \beta, L_{2}\right] \in \tilde{\mathrm{Pic}}^{f}(U)$ such that

$$
\rho(U)\left(\left[L_{1}, \beta, L_{2}\right],\left[\left(E_{1}, \tau_{1}\right), \nu,\left(E_{2}, \tau_{2}\right)\right]\right)=\left[\left(E_{1}^{\prime}, \tau_{1}^{\prime}\right), \nu^{\prime},\left(E_{2}^{\prime}, \tau_{2}^{\prime}\right)\right] .
$$

This proves (1).

Since $S$ is connected, $A_{1}$ and $A_{2}$ have constant rank say $n^{2}$ and $k^{2}$. Let $j: U \rightarrow S$ be an étale map. Then $j^{*} A_{1}$ and $j^{*} A_{2}$ are Azumaya algebras on $U$ of rank $n^{2}$ and $k^{2}$. By Proposition IV.2.1(d) of [12], there exist an étale covering $\left\{b_{i}: U_{i} \rightarrow U\right\}$ such that

$$
\begin{aligned}
& \tau_{1}: M_{n}\left(\mathcal{O}_{U_{i}}\right) \cong b_{i}^{*} j^{*} A_{1} \\
& \tau_{2}: M_{k}\left(\mathcal{O}_{U_{i}}\right) \cong b_{i}^{*} j^{*} A_{2}
\end{aligned}
$$

for each $i$. This implies that $\left[\left(\mathcal{O}_{U_{i}}^{\oplus n}, \tau_{1}\right), \nu,\left(\mathcal{O}_{U_{i}}^{\oplus k}, \tau_{2}\right)\right] \in\left[G_{A}\right]\left(U_{i}\right)$ for all $i$. Here $\nu$ is the obvious isomorphism. Hence $\left[G_{A}\right]\left(U_{i}\right) \neq \emptyset$ for all $i$. This proves $(2)$.

Remark 4.4. Note that the action $\rho(U)$ is simply transitive without the assumption $S$ being connected.

Lemma 4.5. Let $\psi: A:=\left(A_{1}, \alpha, A_{2}\right) \rightarrow B:=\left(B_{1}, \beta, B_{2}\right)$ be a morphism in $\tilde{A} z\left(f^{*}\right)$. Then the induced map $\left[G_{A}\right] \rightarrow\left[G_{B}\right]$ is an isomorphism as $\tilde{\mathrm{Pic}}^{f}$-torsors.

Proof. Since $\alpha$ and $\beta$ are morphisms in $\tilde{A} z(X), \alpha=[(P, u, Q)]$ and $\beta=[(R, v, T)]$. We have $u: f^{*} A_{1} \otimes \operatorname{End}(P) \cong f^{*} A_{2} \otimes \operatorname{End}(Q)$ and $v: f^{*} B_{1} \otimes \operatorname{End}(R) \cong f^{*} B_{2} \otimes \operatorname{End}(T)$. Write $\psi=\left(\psi_{1}, \psi_{2}\right)$, where $\psi_{1}:=\left[\left(P_{1}, u_{1}, Q_{1}\right)\right]$ and $\psi_{2}:=\left[\left(R_{1}, v_{1}, T_{1}\right)\right]$ are morphisms in $\tilde{A} z(S)$. In view of Remark 2.1, we prefer to write $\alpha=[(P, Q)], \beta=[(R, T)], \psi_{1}=$ $\left[\left(P_{1}, Q_{1}\right)\right]$ and $\psi_{2}=\left[\left(R_{1}, T_{1}\right)\right]$. Since $\psi$ is a morphism, the following diagram

$$
\begin{array}{r}
f^{*} A_{1} \stackrel{[(P, Q)]}{\longrightarrow} f^{*} A_{2} \\
{\left[\left(f^{*} P_{1}, f^{*} Q_{1}\right)\right] \downarrow \quad\left[\left(f^{*} R_{1}, f^{*} T_{1}\right)\right] \downarrow} \\
f^{*} B_{1} \stackrel{[(R, T)]}{\longrightarrow} f^{*} B_{2}
\end{array}
$$

commutes, i.e.,

$$
\left[\left(f^{*} R_{1} \otimes P, f^{*} T_{1} \otimes Q\right)\right]=\left[\left(R \otimes f^{*} P_{1}, T \otimes f^{*} Q_{1}\right)\right] .
$$

This means that there exist self dual locally free $\mathcal{O}_{X}$-modules $H_{1}$ and $H_{2}$ of finite rank over $X$ such that

$$
f^{*} R_{1} \otimes P \otimes H_{1} \cong R \otimes f^{*} P_{1} \otimes H_{2}, f^{*} T_{1} \otimes Q \otimes H_{1} \cong T \otimes f^{*} Q_{1} \otimes H_{2} .
$$

Let $\phi_{1}=\left(\left[\left(P_{1}, \mathcal{O}_{S}\right)\right],\left[\left(R_{1}, \mathcal{O}_{S}\right)\right]\right)$ and $\phi_{2}=\left(\left[\left(Q_{1}, \mathcal{O}_{S}\right)\right],\left[\left(T_{1}, \mathcal{O}_{S}\right)\right]\right)$. 
We claim that

$$
\begin{aligned}
& \phi_{1}: A:=\left(A_{1}, \alpha, A_{2}\right) \rightarrow A^{\prime}:=\left(A_{1} \otimes \operatorname{End}\left(P_{1}\right), \alpha^{\prime}, A_{2} \otimes \operatorname{End}\left(R_{1}\right)\right), \\
& \phi_{2}: B:=\left(B_{1}, \beta, B_{2}\right) \rightarrow B^{\prime}:=\left(B_{1} \otimes \operatorname{End}\left(Q_{1}\right), \beta^{\prime}, B_{2} \otimes \operatorname{End}\left(T_{1}\right)\right)
\end{aligned}
$$

both are morphisms in $\tilde{A} z\left(f^{*}\right)$, where $\alpha^{\prime}:=\left[\left(P \otimes f^{*} R_{1}, Q \otimes f^{*} P_{1}\right)\right]$ and $\beta^{\prime}:=[(R \otimes$ $\left.\left.f^{*} T_{1}, T \otimes f^{*} Q_{1}\right)\right]$. Clearly, $\left[\left(P_{1}, \mathcal{O}_{S}\right)\right]$ and $\left.\left[\left(R_{1}, \mathcal{O}_{S}\right)\right]\right)$ both are morphisms in $\tilde{A} z(S)$. Note that $\left(P \otimes f^{*} R_{1} \otimes f^{*} P_{1}, Q \otimes f^{*} P_{1}\right) \sim\left(P \otimes f^{*} R_{1}, Q\right)$ in $\tilde{\Delta}\left(f^{*} A_{1}, f^{*} A_{2} \otimes f^{*} \operatorname{End}\left(R_{1}\right)\right)$. Thus the following diagram

$$
\begin{array}{ccc}
f^{*} A_{1} & \stackrel{[(P, Q)]}{\longrightarrow} & f^{*} A_{2} \\
\left.\left[\left(f^{*} P_{1}, \mathcal{O}_{X}\right)\right]\right\rfloor & \left.\left[\left(f^{*} R_{1}, \mathcal{O}_{X}\right)\right]\right\rfloor \\
f^{*} A_{1} \otimes f^{*} \operatorname{End}\left(P_{1}\right) \stackrel{\left[\left(P \otimes f^{*} R_{1}, Q \otimes f^{*} P_{1}\right)\right]}{\longrightarrow} f^{*} A_{2} \otimes f^{*} \operatorname{End}\left(R_{1}\right)
\end{array}
$$

commutes. Therefore, $\phi_{1}$ is a morphism in $\tilde{A} z\left(f^{*}\right)$. Similarly for $\phi_{2}$. Hence the cliam.

Moreover $\alpha^{\prime} \sim \beta^{\prime}$, because there exist self dual $\mathcal{O}_{X}$-modules $f^{*} T_{1} \otimes H_{1}, f^{*} P_{1} \otimes H_{2}$ such that (by using (4.7))

$P \otimes f^{*} R_{1} \otimes f^{*} T_{1} \otimes H_{1} \cong R \otimes f^{*} T_{1} \otimes f^{*} P_{1} \otimes H_{2}, Q \otimes f^{*} P_{1} \otimes f^{*} P_{1} \otimes H_{2} \cong T \otimes f^{*} Q_{1} \otimes f^{*} P_{1} \otimes H_{2}$.

This implies that $\left(\left[\left(\mathcal{O}_{S}, \mathcal{O}_{S}\right)\right],\left[\left(\mathcal{O}_{S}, \mathcal{O}_{S}\right)\right]\right)$ defines a morphism $A^{\prime} \rightarrow B^{\prime}$ in $\tilde{A} z\left(f^{*}\right)$.

Now for an étale map $j: U \rightarrow S, \phi_{1}$ induces a $\tilde{P i c}^{f}(U)$-linear map $\left[G_{A}\right](U) \rightarrow\left[G_{A^{\prime}}\right](U)$ by sending

$$
\left[\left(E_{1}, \tau_{1}\right), \nu,\left(E_{2}, \tau_{2}\right)\right] \text { to }\left[\left(E_{1} \otimes j^{*} P_{1}, \tau_{1}^{\prime}\right), \nu^{\prime},\left(E_{2} \otimes j^{*} R_{1}, \tau_{2}^{\prime}\right)\right],
$$

where $\tau_{1}^{\prime}: \operatorname{End}\left(E_{1} \otimes j^{*} P_{1}\right) \cong j^{*}\left(A_{1} \otimes \operatorname{End}\left(P_{1}\right)\right), \tau_{2}^{\prime}: \operatorname{End}\left(E_{2} \otimes j^{*} R_{1}\right) \cong j^{*}\left(A_{2} \otimes \operatorname{End}\left(R_{1}\right)\right)$ and $\nu^{\prime}: f_{U}^{*}\left(E_{1} \otimes j^{*} P_{1}\right) \otimes\left(f_{U}^{*}\left(E_{2} \otimes j^{*} R_{1}\right)\right)^{\vee} \cong\left(f_{U}^{*}\left(E_{1} \otimes j^{*} P_{1}\right)\right)^{\vee} \otimes f_{U}^{*}\left(E_{2} \otimes j^{*} R_{1}\right)$ (for $\nu^{\prime}$, we use $\nu$ and the fact that $P_{1}^{\vee} \cong P_{1}$ and $\left.R_{1}^{\vee} \cong R_{1}\right)$. Note that $\tilde{\mathrm{Pic}}^{f}(U)$ acts on both $\left[G_{A}\right](U)$, $\left[G_{A^{\prime}}\right](U)$ simply transitively (see Remark 4.4). Thus, we get $\left[G_{A}\right](U) \cong\left[G_{A^{\prime}}\right](U)$ as sets. Therefore $\left[G_{A}\right] \cong\left[G_{A^{\prime}}\right]$ as Pic $\tilde{\mathrm{ic}}^{f}$-torsors. Similarly for $\phi_{2}$, i.e., $\left[G_{B}\right] \cong\left[G_{B^{\prime}}\right]$. Clearly, $\left[G_{A^{\prime}}\right] \cong\left[G_{B^{\prime}}\right]$ because $\left(\left[\left(\mathcal{O}_{S}, \mathcal{O}_{S}\right)\right],\left[\left(\mathcal{O}_{S}, \mathcal{O}_{S}\right)\right]\right)$ defines a morphism $A^{\prime} \rightarrow B^{\prime}$ in $\tilde{A} z\left(f^{*}\right)$. Hence the result.

Remark 4.6. We do not know whether the Lemma 4.5 holds or not whenever $\psi$ is a morphism in $A z\left(f^{*}\right)$.

Presheaf contracted products. Let $\mathcal{G}$ be a presheaf of groups. Let $\mathcal{F}_{1}$ and $\mathcal{F}_{2}$ be two presheaves of sets on a site $\mathcal{C}$. Suppose that $\mathcal{F}_{1}$ (resp. $\mathcal{F}_{2}$ ) has a right (resp. left) $\mathcal{G}$ action. We can consider the left action of $\mathcal{G}$ on the product given for every object $X$ by:

$$
\left(\mathcal{G} \times \mathcal{F}_{1} \times \mathcal{F}_{2}\right)(X) \rightarrow\left(\mathcal{F}_{1} \times \mathcal{F}_{2}\right)(X),(g, x, y) \mapsto\left(x g^{-1}, g y\right) .
$$


Then the presheaf contracted product of $\mathcal{F}_{1}$ and $\mathcal{F}_{2}$ to be the presheaf defined as the quotient by the action of $\mathcal{G}$ :

$$
X \mapsto \mathcal{F}_{1}(X) \times \mathcal{F}_{2}(X) / \sim
$$

i.e., the quotient by the equivalence relation define by $(x g, y) \sim(x, g y)$ for all $g \in \mathcal{G}(X)$. It is denoted by $\mathcal{F}_{1} \Pi^{\mathcal{G}} \mathcal{F}_{2}$. We write $[x, y]$ for the element of $\mathcal{F}_{1} \Pi^{\mathcal{G}} \mathcal{F}_{2}(X)$, where $X \in o b(\mathcal{C})$, $x \in \mathcal{F}_{1}(X)$ and $y \in \mathcal{F}_{2}(X)$. For every $X \in o b(\mathcal{C})$, the left action of $\mathcal{G}$ on the contracted product is given by

$$
\left(\mathcal{G} \times \mathcal{F}_{1} \Pi^{\mathcal{G}} \mathcal{F}_{2}\right)(X) \rightarrow\left(\mathcal{F}_{1} \Pi^{\mathcal{G}} \mathcal{F}_{2}\right)(X),(g,[x, y]) \mapsto[g . x, y] .
$$

Assume that $\mathcal{G}$ is a presheaf of abelian groups. Then there is no distinction between left and right $\mathcal{G}$-torsors. Let $\operatorname{Tors}(S, \mathcal{G})$ denotes the set of isomorphism classes of $\mathcal{G}$ torsors. It is well known that the $\operatorname{set} \operatorname{Tors}(S, \mathcal{G})$ has an abelian group structure under the operation presheaf contracted product with identity $\mathcal{G}$ and the inverse of $\mathcal{F}$ is $\mathcal{F}$ itself with the $\mathcal{G}$ action $(g, x) \mapsto g^{-1} \cdot x$.

Lemma 4.7. (1) Let $A:=\left(A_{1}, \alpha, A_{2}\right), A^{\prime}:=\left(A_{1}^{\prime}, \alpha^{\prime}, A_{2}^{\prime}\right)$ be in $\tilde{A} z\left(f^{*}\right)$. Write $A \otimes$ $A^{\prime}:=\left(A_{1} \otimes A_{1}^{\prime}, \alpha \otimes \alpha^{\prime}, A_{2} \otimes A_{2}^{\prime}\right)$. Then $\left[G_{A \otimes A^{\prime}}\right] \cong\left[G_{A}\right] \Pi^{\tilde{P} i c^{f}}\left[G_{A^{\prime}}\right]$ as $\tilde{\mathrm{Pic}}^{f}$-torsors.

(2) Let $A:=\left(A_{1}, \alpha, A_{2}\right), B:=\left(A_{2}, \beta, A_{3}\right)$ be in $\tilde{A} z\left(f^{*}\right)$. Write $B \circ A:=\left(A_{1}, \beta \alpha, A_{3}\right)$. Then $\left[G_{B \circ A}\right] \cong\left[G_{A}\right] \Pi^{\tilde{P} i c^{f}}\left[G_{B}\right]$ as $\mathrm{Pic}^{f}$-torsors.

Proof. (1) For an étale map $U \rightarrow S$, we have a $\tilde{\mathrm{Pic}}^{f}(U)$-linear map

$$
\left[G_{A}\right](U) \times\left[G_{A^{\prime}}\right](U) / \sim \rightarrow\left[G_{A \otimes A^{\prime}}\right](U),
$$

sending

$\left[\left[\left(E_{1}, \tau_{1}\right), \nu,\left(E_{2}, \tau_{2}\right)\right],\left[\left(E_{1}^{\prime}, \tau_{1}^{\prime}\right), \nu^{\prime},\left(E_{2}^{\prime}, \tau_{2}^{\prime}\right)\right]\right]$ to $\left[\left[\left(E_{1} \otimes E_{1}^{\prime}, \tau_{1} \otimes \tau_{1}^{\prime}\right), \nu \otimes \nu^{\prime},\left(E_{2} \otimes E_{2}^{\prime}, \tau_{2} \otimes \tau_{2}^{\prime}\right)\right]\right]$.

Since $\tilde{\mathrm{Pic}}^{f}(U)$ acts simply transitively on $\left[G_{A}\right](U) \times\left[G_{A^{\prime}}\right](U) / \sim$ and $\left[G_{A \otimes A^{\prime}}\right](U)$, we get $\left[G_{A}\right](U) \times\left[G_{A^{\prime}}\right](U) / \sim \cong\left[G_{A \otimes A^{\prime}}\right](U)$ as sets. Therefore $\left[G_{A \otimes A^{\prime}}\right] \cong\left[G_{A}\right] \Pi^{\tilde{P} i c^{f}}\left[G_{A^{\prime}}\right]$ as $\tilde{\mathrm{Pic}}^{f}$-torsors.

(2) Let $\left[\left(E_{1}, \tau_{1}\right), \nu,\left(E_{2}, \tau_{2}\right)\right] \in\left[G_{A}\right](U)$ and $\left[\left(E_{2}^{\prime}, \tau_{2}^{\prime}\right), \nu^{\prime},\left(E_{3}, \tau_{3}\right)\right] \in\left[G_{B}\right](U)$. Since $\left(E_{2}, \tau_{2}\right)$ and $\left(E_{2}^{\prime}, \tau_{2}^{\prime}\right) \in F_{A_{2}}(U)$, there exists a unique line bundle $L$ such that $E_{2}^{\prime} \cong E_{2} \otimes L$ (see Lemma 4.3 of [6]). The map $\nu \otimes \nu^{\prime}$ gives an isomorphism

$$
f_{U}^{*}\left(E_{1} \otimes L\right) \otimes\left(f_{U}^{*} E_{3}\right)^{\vee} \otimes \operatorname{End}\left(f_{U}^{*} E_{2}\right) \cong\left(f_{U}^{*}\left(E_{1} \otimes L\right)\right)^{\vee} \otimes f_{U}^{*} E_{3} \otimes \operatorname{End}\left(f_{U}^{*} E_{2}\right)
$$

of $\mathcal{O}_{X \times S} U^{-m o d u l e s . ~ T h e n ~ b y ~ a p p l y i n g ~ L e m m a ~ 4.1, ~ w e ~ g e t ~ a n ~} \mathcal{O}_{X \times{ }_{S} U^{-}}$,module isomorphism $\chi: f_{U}^{*}\left(E_{1} \otimes L\right) \otimes\left(f_{U}^{*} E_{3}\right)^{\vee} \cong\left(f_{U}^{*}\left(E_{1} \otimes L\right)\right)^{\vee} \otimes f_{U}^{*} E_{3}$. This shows that $\left[\left(E_{1} \otimes\right.\right.$ $\left.\left.L, \tau_{1} a_{1}\right), \chi,\left(E_{3}, \tau_{3}\right)\right] \in\left[G_{B \circ A}\right](U)$, where $a_{1}: \operatorname{End}\left(E_{1} \otimes L\right) \cong \operatorname{End}\left(E_{1}\right)$. Now, we have a $\tilde{P i c}^{f}(U)$-linear map

$$
\left[G_{A}\right](U) \times\left[G_{B}\right](U) / \sim \rightarrow\left[G_{B \circ A}\right](U)
$$


sending $\left[\left[\left(E_{1}, \tau_{1}\right), \nu,\left(E_{2}, \tau_{2}\right)\right],\left[\left(E_{1}^{\prime}, \tau_{1}^{\prime}\right), \nu^{\prime},\left(E_{3}, \tau_{3}\right)\right]\right]$ to $\left[\left[\left(E_{1} \otimes L, \tau_{1} a_{1}\right), \chi,\left(E_{3}, \tau_{3}\right)\right]\right]$. The rest of the arguments similar to (1). Hence the result.

By Lemma 4.5, we obtain a well-define map

$\left\{\right.$ The set of isomorphism classes of objects in $\left.A \tilde{z}\left(f^{*}\right)\right\} \rightarrow \operatorname{Tors}\left(S, \tilde{\mathrm{Pic}}^{f}\right),[A] \mapsto\left[G_{A}\right]$.

In fact, this induces a well-define group homomorphism

$$
\omega: \tilde{\operatorname{Br}}(f) \rightarrow \operatorname{Tors}\left(S, \operatorname{Pic}^{f}\right)
$$

by Lemma 4.7. Moreover, $\omega$ is a natural map (see below).

Lemma 4.8. The map $\omega$ is natural.

Proof. Given a commutative diagram

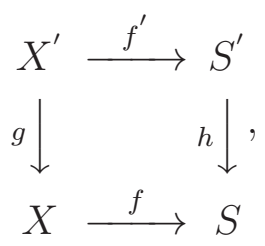

we want to show that the following diagram



is commutative. Here $h^{*}\left(\left[\left(A_{1}, \alpha, A_{2}\right)\right]\right)=\left[\left(h^{*} A_{1}, h^{*} \alpha, h^{*} A_{2}\right)\right]$ and Tors $(h)$ is defined as follows. For an étale map $V \stackrel{i}{\rightarrow} S^{\prime},\left(h^{-1} \mathcal{G}\right)(V):=\lim _{\longrightarrow} \mathcal{G}(U)$, where $\mathcal{G} \in \operatorname{Tors}\left(S, \tilde{P i c}^{f}\right)$ and the direct limit is over the commutative diagrams

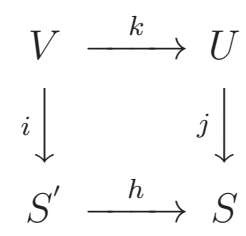

with $j$ étale. Now, for such a diagram, we obtain a natural group homomorphism

$$
\tilde{\operatorname{Pic}}^{f}(U) \rightarrow \tilde{\operatorname{Pic}}^{f^{\prime}}(V),\left[L_{1}, a, L_{2}\right] \mapsto\left[k^{*} L_{1}, \varepsilon^{*} a, k^{*} L_{2}\right] .
$$

Here $\varepsilon$ is the unique map $X^{\prime} \times{ }_{S^{\prime}} V \rightarrow X \times{ }_{S} U$ compatible with the maps $g, h$ and $k$. By the universal property of direct limits, there is a unique homomorphism $h^{-1} \tilde{\mathrm{Pic}}^{f} \rightarrow \tilde{\mathrm{Pic}}^{f^{\prime}}$. Then $\operatorname{Tors}(h)(\mathcal{G})=h^{-1} \mathcal{G} \Pi^{h^{-1} \tilde{\mathrm{Pic}}^{f}} \tilde{\mathrm{Pic}}^{f^{\prime}}$. 
More explicitly, we have to show that there is an isomorphism

$$
h^{-1}\left[G_{\left(A_{1}, \alpha, A_{2}\right)}\right] \Pi^{h^{-1} \tilde{\mathrm{Pic}}^{f}} \tilde{\mathrm{Pic}}^{f^{\prime}} \cong\left[G_{\left(h^{*} A_{1}, h^{*} \alpha, h^{*} A_{2}\right)}\right]
$$

of $\tilde{\mathrm{Pic}}^{f^{\prime}}$-torsors. Note that we have a $\tilde{\mathrm{Pic}}^{f}(U)$-linear map

$$
\left[G_{\left(A_{1}, \alpha, A_{2}\right)}\right](U) \rightarrow\left[G_{\left(h^{*} A_{1}, h^{*} \alpha, h^{*} A_{2}\right)}\right](V),
$$

sending $\left[\left(E_{1}, \tau_{1}\right), \nu,\left(E_{2}, \tau_{2}\right)\right]$ to $\left[\left(k^{*} E_{1}, k^{*} \tau_{1}\right), \varepsilon^{*} \nu,\left(k^{*} E_{2}, k^{*} \tau_{2}\right)\right]$. Therefore, we deduce a $\tilde{\mathrm{Pic}}^{f^{\prime}}(V)$-linear map

$$
h^{-1}\left[G_{\left(A_{1}, \alpha, A_{2}\right)}\right](V) \times \tilde{\mathrm{Pic}}^{f^{\prime}}(V) / \sim \rightarrow\left[G_{\left(h^{*} A_{1}, h^{*} \alpha, h^{*} A_{2}\right)}\right](V)
$$

by passing to the limit over such all such diagrams. Since $\tilde{\mathrm{Pic}}^{f^{\prime}}(V)$ acts simply transitively on both sides, we get the result.

Now, by using the maps $\Upsilon$ (see (2.2) ) and $\Psi$ (see (4.4)), we define a natural group homomorphism $\operatorname{Br}(f) \rightarrow \operatorname{Tors}\left(S, \operatorname{Pic}^{f}\right)$ as follows

$$
\operatorname{Br}(f) \stackrel{\Upsilon}{\rightarrow} \tilde{\operatorname{Br}}(f) \stackrel{\omega}{\rightarrow} \operatorname{Tors}\left(S, \tilde{P i c}^{f}\right) \stackrel{\operatorname{Tors}(S, \Psi)}{\longrightarrow} \operatorname{Tors}\left(S, \operatorname{Pic}^{f}\right)
$$

where $\operatorname{Tors}(S, \Psi)$ is the natural induced map sending any $\mathrm{Pic}^{f}$-torsor $\mathcal{G}$ to $\mathcal{G} \Pi^{\mathrm{Pic}^{f}} \operatorname{Pic}^{f}$.

Lemma 4.9. There is a natural group homomorphism $\zeta: \operatorname{Tors}(S, \mathcal{G}) \rightarrow \check{H}_{\text {et }}^{1}(S, \mathcal{G})$, where $\mathcal{G}$ is a presheaf of abelian groups and $\check{H}_{\text {et }}^{1}(S, \mathcal{G})$ denotes the first étale Čech cohomology group associated to $\mathcal{G}$.

Proof. For the map $\zeta$, we refer to section 11 of [13] or Proposition 4.6 of [12]. The only difference is that we are defining the map here for presheaf torsors. However, we observe that the sheaf properties have not been used in [13] to define such a map. Hence, one can define such map in a similar way for presheaf torsors as well.

By definition $\check{H}_{e t}^{1}(S, \mathcal{G})=\underline{\lim }_{\mathcal{U}} \check{H}_{e t}^{1}(\mathcal{U}, \mathcal{G})$, where the limit is taken over all covering $\mathcal{U}=\left\{U_{i} \rightarrow S\right\}$. Write $U_{i j}$ for $U_{i} \times{ }_{S} U_{j}$. Let $\mathcal{F}_{1}, \mathcal{F}_{2} \in \operatorname{Tors}(S, \mathcal{G})$. Then for some covering $\mathcal{U}=\left\{U_{i} \rightarrow S\right\}, \zeta\left(\mathcal{F}_{1}\right)=\left(g_{i j}\right)_{(i, j) \in I \times I}$ and $\zeta\left(\mathcal{F}_{2}\right)=\left(h_{i j}\right)_{(i, j) \in I \times I}$, where $g_{i j}, h_{i j} \in \mathcal{G}\left(U_{i j}\right)$. Note that $\left(g_{i j}\right)_{(i, j) \in I \times I}$ and $\left(h_{i j}\right)_{(i, j) \in I \times I}$ satisfy the following properties: $\left.g_{i, j} \cdot x_{i}\right|_{U_{i j}}=\left.x_{j}\right|_{U_{i j}}$ and $\left.h_{i, j} \cdot y_{i}\right|_{U_{i j}}=\left.y_{j}\right|_{U_{i j}}$, where $x_{i} \in \mathcal{F}_{1}\left(U_{i}\right), y_{i} \in \mathcal{F}_{2}\left(U_{i}\right)$. Recall that $\left(\mathcal{F}_{1} \Pi \mathcal{G}^{\mathcal{G}} \mathcal{F}_{2}\right)\left(U_{i}\right)$ consists of elements of the form $\left[x_{i}, y_{i}\right]$ with $x_{i} \in \mathcal{F}_{1}\left(U_{i}\right), y_{i} \in \mathcal{F}_{2}\left(U_{i}\right)$. We have

$$
g_{i j} h_{i j} \cdot\left[\left.x_{i}\right|_{U_{i j}},\left.y_{i}\right|_{U_{i j}}\right]=\left[\left.g_{i j} h_{i j} \cdot x_{i}\right|_{U_{i j}},\left.y_{i}\right|_{U_{i j}}\right]=\left[\left.g_{i j} \cdot x_{i}\right|_{U_{i j}}, h_{i j} .\left.y_{i}\right|_{U_{i j}}\right]=\left[\left.x_{j}\right|_{U_{i j}},\left.y_{j}\right|_{U_{i j}}\right] .
$$

This implies that $\left(g_{i j} h_{i j}\right)_{(i, j) \in I \times I}$ defines an element of $\check{H}_{\text {et }}^{1}(\mathcal{U}, \mathcal{G})$. Therefore $\zeta\left(\mathcal{F}_{1} \Pi^{\mathcal{G}} \mathcal{F}_{2}\right)=$ $\left(g_{i j} h_{i j}\right)_{(i, j) \in I \times I}=\zeta\left(\mathcal{F}_{1}\right) \zeta\left(\mathcal{F}_{2}\right)$ and hence $\zeta$ is a group homomorphism.

Remark 4.10. If $\mathcal{G}$ is a sheaf of abelian groups then the map $\zeta$ is an isomorphism (see Proposition 11.1 of [13]). 
Lemma 4.11. Let $\mathcal{P} i c_{e t}^{f}$ be the étale sheafification of the presheaf $\operatorname{Pic}^{f}$ on $S_{\text {et }}$. Then

$$
\left(f_{*} \mathcal{O}_{X}^{\times} / \mathcal{O}_{S}^{\times}\right)_{e t} \cong \mathcal{P} i c_{e t}^{f}
$$

Proof. For each étale $U \rightarrow S$, there is a natural map (using (4.3))

$$
\Gamma\left(\tilde{X}, \mathcal{O}_{\tilde{X}}\right)^{\times} / \Gamma\left(U, \mathcal{O}_{U}\right)^{\times} \rightarrow \operatorname{Pic}^{f}(U) \rightarrow \mathcal{P i c}^{f}(U)
$$

Here $\tilde{X}$ stands for $X \times{ }_{S} U$. Then by the universal property of sheafification, we get a unique map $\left(f_{*} \mathcal{O}_{X}^{\times} / \mathcal{O}_{S}^{\times}\right)_{e t} \rightarrow \mathcal{P} i c_{e t}^{f}$ of sheaves. Since Pic vanishes for a strictly hensel local ring, $\left(\left(f_{*} \mathcal{O}_{X}^{\times} / \mathcal{O}_{S}^{\times}\right)_{e t}\right)_{\bar{s}} \cong\left(\mathcal{P} i c_{e t}^{f}\right)_{\bar{s}}$ for all geometric point $\bar{s}$ of $S$ by the sequence (4.3). Therefore, $\left(f_{*} \mathcal{O}_{X}^{\times} / \mathcal{O}_{S}^{\times}\right)_{e t} \cong \mathcal{P} i c_{e t}^{f}$.

Theorem 4.12. Let $f: X \rightarrow S$ be a faithful affine map of noetherian schemes. Then there is a natural group homomorphism $\theta: \operatorname{Br}(f) \rightarrow \operatorname{Br}^{\prime}(f):=H_{e t}^{1}\left(S, f_{*} \mathcal{O}_{X}^{\times} / \mathcal{O}_{S}^{\times}\right)$.

Proof. Since $S$ is noetherian, we can write $S=\sqcup_{i=1}^{n} S_{i}$, where $S_{i}$ 's are connected components of $S$. Then $X=\sqcup_{i=1}^{n} X \times_{S} S_{i}$ and $f=\sqcup_{i=1}^{n} f_{i}$, where $f_{i}: X \times_{S} S_{i} \rightarrow S_{i}$. For each $\eta_{i}: f_{i} \rightarrow f$, we get an induced map $\gamma_{i}: \operatorname{Br}(f) \rightarrow \operatorname{Br}\left(f_{i}\right)$. Therefore, $\gamma=\left(\gamma_{i}\right)$ defines a map $\operatorname{Br}(f) \rightarrow \operatorname{Br}\left(f_{1}\right) \times \cdots \times \operatorname{Br}\left(f_{n}\right)$. Moreover, the cohomology $H^{i}$ commute with the finite disjoint union. So, we may assume that $S$ is connected. Now, we note that the following two well-known facts.

(1) Given a presheaf $\mathcal{F}$ of abelian groups on $S_{\text {et }}$ and the sheafification $\mathcal{F}^{+}$, there is always a natural map

$$
\check{H}_{e t}^{i}(S, \mathcal{F}) \rightarrow \check{H}_{e t}^{i}\left(S, \mathcal{F}^{+}\right)
$$

(2) For all abelian sheaf $\mathcal{F}$, the natural map

$$
\check{H}_{e t}^{1}(S, \mathcal{F}) \rightarrow H_{e t}^{1}(S, \mathcal{F})
$$

is an isomorphism.

The above two facts together with Lemmas 4.9 and 4.11 allow us to define the desired map $\theta$ as the composition of the following maps (see also (4.9))

$$
\begin{aligned}
& \operatorname{Br}(f) \stackrel{\Upsilon}{\rightarrow} \tilde{\operatorname{Br}}(f) \stackrel{\omega}{\rightarrow} \operatorname{Tors}\left(S, \tilde{\mathrm{Pic}}^{f}\right) \stackrel{\operatorname{Tors}(S, \Psi)}{\longrightarrow} \operatorname{Tors}\left(S, \operatorname{Pic}^{f}\right) \stackrel{\zeta}{\rightarrow} \check{H}_{\mathrm{et}}^{1}\left(S, \operatorname{Pic}^{f}\right) \rightarrow \check{H}_{\mathrm{et}}^{1}\left(S, \mathcal{P i c}^{f}\right) \\
& \cong \check{H}_{e t}^{1}\left(S, f_{*} \mathcal{O}_{X}^{\times} / \mathcal{O}_{S}^{\times}\right) \stackrel{\cong}{\rightarrow} H_{e t}^{1}\left(S, f_{*} \mathcal{O}_{X}^{\times} / \mathcal{O}_{S}^{\times}\right) .
\end{aligned}
$$

Remark 4.13. The map $\theta$ is not injective in general (see Example 3.3). 


\section{The Relative Brauer group of subintegral extensions}

In this section, we study the relative Brauer group $\operatorname{Br}(f)$ in the case when $f$ is a subintegral extension. Some details pertaining to subintegral extensions can be found in [21].

Theorem 5.1. Let $f: A \hookrightarrow B$ be a subintegral extension of noetherian $\mathbb{Q}$-algebras. Then the following are true:

(1) the natural map $f^{*}: \operatorname{Br}(A) \rightarrow \operatorname{Br}(B)$ is an isomorphism.

(2) $\operatorname{Br}(f)=0$.

Proof. (1) Since $f$ is subintegral, $B=\cup_{\lambda} B_{\lambda}$ where each $B_{\lambda}$ can be obtained by finite succession of elementary subintegral extension (i.e. $A \subset A[b]$ such that $b^{2}, b^{3} \in A$ ) (see [21]). In other words, $f=\cup_{\lambda} f_{\lambda}$ where $f_{\lambda}: A \hookrightarrow B_{\lambda}$ is a finite map. For each $\lambda$, we have the following two exact sequences

$$
0 \rightarrow \operatorname{ker}\left(f_{\lambda}^{*}\right) \rightarrow \operatorname{Br}(A) \stackrel{f_{\lambda}^{*}}{\rightarrow} \operatorname{Br}\left(B_{\lambda}\right), \operatorname{Br}(A) \stackrel{f_{\lambda}^{*}}{\rightarrow} \operatorname{Br}\left(B_{\lambda}\right) \rightarrow \operatorname{coker}\left(f_{\lambda}^{*}\right) \rightarrow 0 .
$$

As $\mathrm{Br}$ commutes with filtered limit of rings, $\operatorname{Br}(B) \cong \lim _{\lambda} \operatorname{Br}\left(B_{\lambda}\right)$. Thus we get $\operatorname{ker}\left(f^{*}\right) \cong \lim _{\lambda} \operatorname{ker}\left(f_{\lambda}^{*}\right)$ and $\operatorname{coker}\left(f^{*}\right) \cong \lim _{\lambda} \operatorname{coker}\left(f_{\lambda}^{*}\right)$. So it is enough to show that $\operatorname{ker}\left(f_{\lambda}^{*}\right)=0=\operatorname{coker}\left(f_{\lambda}^{*}\right)$ for each $\lambda$.

By Proposition 5.4(3) of [17], $H_{e t}^{i}\left(\operatorname{Spec}(A), \mathcal{O}_{A}^{\times}\right) \cong H_{e t}^{i}\left(\operatorname{Spec}\left(B_{\lambda}\right), \mathcal{O}_{B_{\lambda}}^{\times}\right)$for all $i>$ 1 because $f_{\lambda}$ is a finite subintegral extension of $\mathbb{Q}$-algebras. In particular, we get $\operatorname{Br}^{\prime}(A) \cong \operatorname{Br}^{\prime}\left(B_{\lambda}\right)$. Then the torsion subgroups $\operatorname{Br}^{\prime}(A)_{\text {tor }}$ and $\operatorname{Br}^{\prime}\left(B_{\lambda}\right)_{\text {tor }}$ are also isomorphic. Therefore, the assertion follows from the following commutative diagram

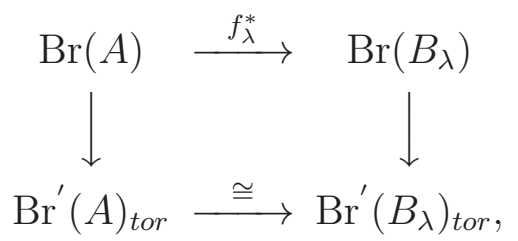

where the vertical maps are isomorphisms by a theorem of Gabber (see [5]).

(2) Since $f$ is subintegral, the map $\operatorname{Pic}(A) \rightarrow \operatorname{Pic}(B)$ is surjective by Proposition 7 of [11. Hence the result by (1) and the sequence (2.1).

Given an extension $f: A \hookrightarrow B$, write ${ }^{+} f$ for the induced map ${ }^{+} A \hookrightarrow B$ where ${ }^{+} A$ is the subintegral closure of $A$ in $B$.

Corollary 5.2. Let $f: A \hookrightarrow B$ be an extension of noetherian $\mathbb{Q}$-algebras. Then $\operatorname{Br}(f) \cong$ $\operatorname{Br}\left({ }^{+} f\right)$.

Proof. By comparing Br-Pic sequences (see (2.1)) for $f$ and ${ }^{+} f$, we get the result. 
We say that a faithful affine map $f: X \rightarrow S$ is subintegral if $\mathcal{O}_{S}(U) \rightarrow f_{*} \mathcal{O}_{X}(U)$ is subintegral for all affine open subsets $U$ of $S$ (see Definition 5.1 of [17]).

Remark 5.3. (Cf. Example 6.6 of [19]) Proposition 5.1](2) is not true for non-affine schemes. For example, consider $S=\mathbb{P}_{\mathbb{Q}}^{1}$ and $X=\operatorname{Spec}\left(\mathcal{O}_{\mathrm{B}}\right)$ where $\mathcal{O}_{B}=\mathcal{O}_{S} \oplus \mathcal{O}_{S}(-2)$ with $\mathcal{O}_{S}(-2)$ being a square zero ideal. Then $f: X \rightarrow S$ is a subintegral map and $H=H^{1}\left(\mathbb{P}^{1}, \mathcal{O}_{S}(-2)\right)$ is nonzero. We also have $\operatorname{Pic}(X)=\operatorname{Pic}(S) \oplus H$. Therefore, $\operatorname{Pic}(S) \rightarrow \operatorname{Pic}(X)$ is not surjective. Hence $\operatorname{Br}(f) \neq 0$ by the sequence (2.1).

\section{Relative KummeR's SEQUence}

For a commutative ring $A$, let $\mu_{n}(A):=\operatorname{ker}\left[A^{\times} \stackrel{n}{\rightarrow} A^{\times}\right]$and for a ring extension $f: A \hookrightarrow B$, let $\mu_{n}(f):=\operatorname{ker}[\operatorname{Pic}(f) \stackrel{n}{\rightarrow} \operatorname{Pic}(f)]$. We can identify Pic $(f)$ with $I(f)$, the multiplicative group of invertible $A$-submodules of $B$ by Lemma 1.2 of [19]. Some details related to $I(f)$ can be found in section 2 of [15].

Lemma 6.1. Let $f: A \hookrightarrow B$ be a finite ring extension. Assume that $A$ is a strictly hensel local ring with residue field $k$ and characteristic of $k$ does not divide $n$. Then the following sequences

$$
\begin{aligned}
& 0 \rightarrow \mu_{n}(A) \rightarrow \mu_{n}(B) \rightarrow \mu_{n}(f) \rightarrow 0, \\
& 0 \rightarrow \mu_{n}(f) \rightarrow \operatorname{Pic}(f) \stackrel{n}{\rightarrow} \operatorname{Pic}(f) \rightarrow 0
\end{aligned}
$$

are exact.

Proof. Since $B$ is finite over $A, B$ is finite product of strictly hensel local rings. We know that for a strictly hensel local ring $A$, the sequence

$$
0 \rightarrow \mu_{n}(A) \rightarrow A^{\times} \stackrel{n}{\rightarrow} A^{\times} \rightarrow 0
$$

is exact. Now the result follows from the following commutative diagram

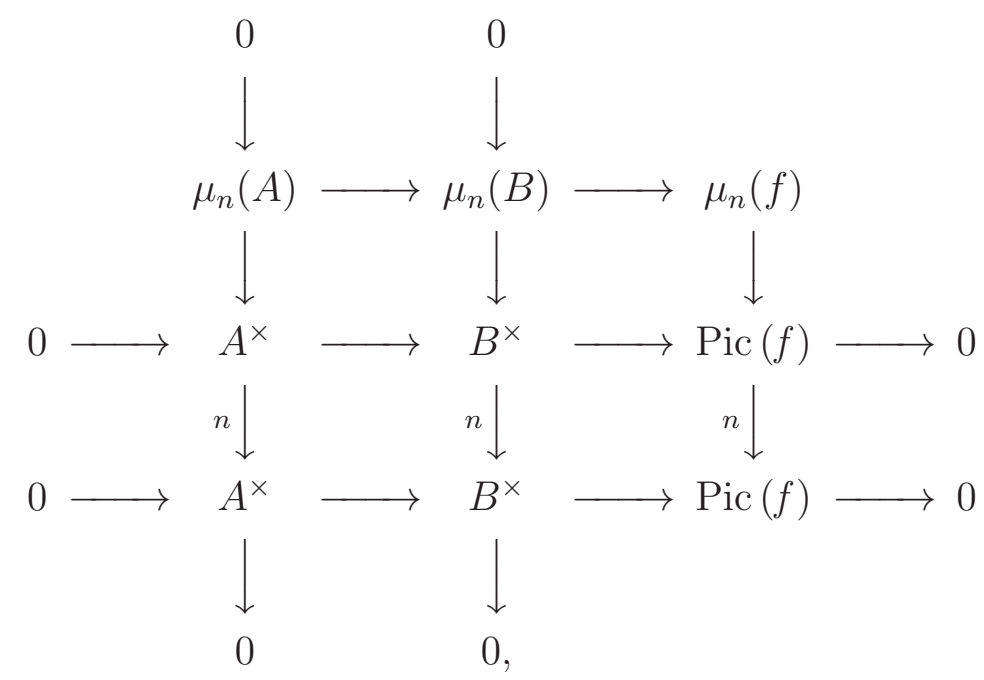


where the left two columns and the bottom two rows are short exact sequences (see (4.3)).

Let $f: X \rightarrow S$ be a faithful affine map of schemes. Recall that $\mathcal{P} i c_{e t}^{f}$ is the étale sheafification of the presheaf $U \mapsto \operatorname{Pic}\left(f_{U}\right)$ on $S_{e t}$, where $f_{U}: X \times{ }_{S} U \rightarrow U$. For notational convenience, we prefer to write $\mathcal{I}_{\text {et }}$ instead of $\mathcal{P} i c_{e t}^{f} \cong\left(f_{*} \mathcal{O}_{X}^{\times} / \mathcal{O}_{S}^{\times}\right)_{e t}$ (see Lemma 4.11).

We write $\mu_{n, X}$ (or simply $\mu_{n}$ ) for the kernel of $\mathcal{O}_{X, e t}^{\times} \stackrel{n}{\rightarrow} \mathcal{O}_{X, e t}^{\times}$. Similarly, $\mu_{n}^{f}$ denotes the kernel of $\mathcal{I}_{\text {et }} \stackrel{n}{\rightarrow} \mathcal{I}_{\text {et }}$.

Proposition 6.2. Let $f: X \rightarrow S$ be a faithful finite map of schemes. Assume that the characteristic of $k(s)$ does not divide $n$ for any $s \in S$. Then the following sequences

$$
\begin{gathered}
0 \rightarrow \mu_{n} \rightarrow f_{*} \mu_{n} \rightarrow \mu_{n}^{f} \rightarrow 0, \\
0 \rightarrow \mu_{n}^{f} \rightarrow \mathcal{I}_{\text {et }} \stackrel{n}{\rightarrow} \mathcal{I}_{\text {et }} \rightarrow 0
\end{gathered}
$$

of étale sheaves on $S_{\text {et }}$ are exact.

Proof. Clear from Lemma 6.1.

From the Proposition 6.2, we obtain the following two long exact sequences

$$
\begin{gathered}
\cdots \rightarrow H_{e t}^{i}\left(S, \mu_{n}\right) \rightarrow H_{e t}^{i}\left(X, \mu_{n}\right) \rightarrow H_{e t}^{i}\left(S, \mu_{n}^{f}\right) \rightarrow H_{e t}^{i+1}\left(S, \mu_{n}\right) \rightarrow \ldots \\
\cdots \rightarrow H_{e t}^{i}\left(S, \mu_{n}^{f}\right) \rightarrow H_{e t}^{i}\left(S, \mathcal{I}_{e t}\right) \stackrel{n}{\rightarrow} H_{e t}^{i}\left(S, \mathcal{I}_{e t}\right) \rightarrow H_{e t}^{i+1}\left(S, \mu_{n}^{f}\right) \rightarrow \ldots
\end{gathered}
$$

For a group $G$, we denote the subgroup of elements of order dividing $n$ in $G$ by ${ }_{n} G$.

Theorem 6.3. Let $f: X \rightarrow S$ be a faithful finite map of schemes. Assume that the characteristic of $k(s)$ does not divide $n$ for any $s \in S$. Then

(1) $H_{e t}^{0}\left(S, \mu_{n}^{f}\right) \cong{ }_{n} \operatorname{Pic}(f)$ and the sequence

$$
0 \rightarrow \operatorname{Pic}(f) \otimes \mathbb{Z} / n \mathbb{Z} \rightarrow H_{e t}^{1}\left(S, \mu_{n}^{f}\right) \rightarrow{ }_{n} H_{e t}^{1}\left(S, \mathcal{I}_{e t}\right) \rightarrow 0
$$

is exact.

(2) if $f: L \hookrightarrow K$ is a finite field extensions and characteristic of $L$ does not divide $n$ then the sequence

$$
0 \rightarrow K^{\times} / L^{\times} \otimes \mathbb{Z} / n \mathbb{Z} \rightarrow H_{e t}^{1}\left(\operatorname{Spec}(L), \mu_{n}^{f}\right) \rightarrow{ }_{n} \operatorname{Br}(K \mid L) \rightarrow 0
$$

is exact. 
Proof. (1) By Lemma 5.4 of [18], $H_{e t}^{0}\left(S, \mathcal{I}_{e t}\right) \cong \operatorname{Pic}(f)$. The long exact sequence (6.2) implies the assertion.

(2) Note that $\operatorname{Pic}(f) \cong K^{\times} / L^{\times}$by (4.3) and $H_{e t}^{1}\left(\operatorname{Spec}(L), f_{*} \mathcal{O}_{K}^{\times} / \mathcal{O}_{L}^{\times}\right) \cong \operatorname{Br}(K \mid L)$ by Lemma 3.1. Hence the result by the short exact sequence (6.3).

Let $\mathcal{P} i c_{z a r}^{f}$ be the Zariski sheaf associated to the presheaf $\operatorname{Pic}^{f}$ on $S_{z a r}$, defined as $\operatorname{Pic}^{f}(U)=\operatorname{Pic}\left(f_{U}\right)$. A proof similar to Lemma 4.11 shows that $\mathcal{P} i c_{z a r}^{f} \cong\left(f_{*} \mathcal{O}_{X}^{\times} / \mathcal{O}_{S}^{\times}\right)_{z a r}$. We write $\mathcal{I}_{z a r}$ for the Zariski sheaf $\mathcal{P} i c_{z a r}^{f} \cong\left(f_{*} \mathcal{O}_{X}^{\times} / \mathcal{O}_{S}^{\times}\right)_{z a r}$.

Theorem 6.4. Let $f: X \rightarrow S$ be a faithful finite map of noetherian schemes over $\mathbb{Q}$.

(1) If $f$ is a subintegral extension of affine schemes then $H_{e t}^{i}\left(S, \mu_{n}^{f}\right)=0$ for $i \geq 0$. Moreover, $H_{e t}^{i}\left(S, \mu_{n}\right) \cong H_{e t}^{i}\left(X, \mu_{n}\right)$ for $i \geq 0$.

(2) If $f$ is subintegral and $S$ is a projective $\mathbb{Q}$-scheme then $H_{\text {et }}^{1}\left(S, \mu_{n}^{f}\right) \cong{ }_{n} H_{\text {et }}^{1}\left(S, \mathcal{I}_{\text {et }}\right)$.

Proof. (1) For a subintegral extension $f: A \hookrightarrow B$ of $\mathbb{Q}$-algebras, we have Pic $(f) \cong B / A$ by Theorem 5.6 of [15] and Theorem 2.3 of [14]. This implies that Pic $(f)$ is a $\mathbb{Q}$-vector space, hence it is a divisible group. Therefore, the map Pic $(f) \stackrel{n}{\rightarrow}$ Pic $(f)$ is bijective. For $i>0, H_{\text {et }}^{i}\left(S, \mathcal{I}_{\text {et }}\right) \cong H_{z a r}^{i}\left(S, \mathcal{I}_{z a r}\right)=0$ by Proposition 5.4 of [17]. Hence, $H_{e t}^{i}\left(S, \mu_{n}^{f}\right)=0$ for $i \geq 0$ by (6.2).

The other part is clear from (6.1).

(2) By Proposition 5.3 of [17], Pic $(f) \cong H_{\text {zar }}^{0}\left(S, f_{*} \mathcal{O}_{X} / \mathcal{O}_{S}\right)$. Note that $f_{*} \mathcal{O}_{X} / \mathcal{O}_{S}$ is coherent. So, Pic $(f)$ is a $\mathbb{Q}$-vector space. Hence the result by the short exact sequence (6.3).

\section{REFERENCES}

[1] B. Auslander, The Brauer Group of a Ringed Space, Journal of Algebra, 4, (1966) 220-273.

[2] M. Auslander and O. Goldman, The Brauer group of a commutative ring. Trans. Amer. Math. Soc. 97, (1960) 367-409.

[3] H. Bass and A. Roy, Lectures on topics in algebraic K-theory, Tata Institute of Fundamental Research Lectures on Mathematics, No. 41, Tata Institute of Fundamental Research, Bombay 1967.

[4] H. Bass, Algebraic K-theory. Benjamin, Newyork, 1968.

[5] A.J. De Jong, A result of Gabber, Preprint, 2003.

[6] J.M Ferncindez Vilaboa, E. Villanueva Novoa and R. Gonzilez Rodriguez, The exact sequence for a monoidal functor between exact categories, Communication in Algebra, 26(9), (1998) 2727-2743.

[7] B. Fein and M. Schacher, Relative Brauer groups. I. J. Reine Angew. Math. 321, (1981) 179-194.

[8] B. Fein and M. Schacher, Kantor. William, Relative Brauer groups. II. J. Reine Angew. Math. 328, (1981) 39-57.

[9] B. Fein and M. Schacher, Relative Brauer groups. III. J. Reine Angew. Math. 335, (1982) 37-39.

[10] J. Giraud, Cohomologie Non Abelienne, Springer, Heidelberg, 1971. 
[11] F. Ischebeck, Subintegral ring extensions and some K-theoretical functors, J. Algebra 121, (1989) 323-338.

[12] J. Milne, Étale Cohomology. Princeton: Princeton University Press 1980.

[13] J. Milne, Lectures on Étale cohomology, http: www.jmilne.org/math/CourseNotes/LEC210.pdf.

[14] L. Reid, L. G. Roberts and B. Singh, Finiteness of subintegrality, in: P.G Goerss and J.F Jardine (eds.) Algebraic K-Theory and Algebraic Topology NATO ASI, Series C, Vol. 407 (Kluwer Academic Publishers, Dordrecht, 1993) 223-227.

[15] L. G. Roberts and B. Singh, Subintegrality, invertible modules and the Picard group, Compositio Math. 85 (1993), 249-279.

[16] Stack Project, Cohomology on sites.

[17] V. Sadhu, On the vanishing of relative negative K-theory, To appear in Journal of Algebra and its applications. https://doi.org/10.1142/S0219498820501522 (Arxiv 1701.09059v3).

[18] V. Sadhu and C. Weibel, Relative Cartier divisors and Laurent polynomial Extensions, Math. Z, 285 (2017), 353-366.

[19] V. Sadhu and C. Weibel, Relative Cartier divisors and K-theory, K-Theory Proceedings of the International Colloquium, Mumbai, 2016, 1-19, Hindustan Book Agency, New Delhi, 2018.

[20] M. Szymik, The Brauer group of Burnside rings, Journal of Algebra 324 (2010) 2589-2593.

[21] R. G. Swan, On Seminormality, J. Algebra 67 (1980), 210-229.

Department of Mathematics, Indian Institute of Science Education and Research, Bhopal, Bhopal Bypass Road, Bhauri, Bhopal-462066, Madhya Pradesh, India

E-mail address: vsadhu@iiserb.ac.in, viveksadhu@gmail.com 